\title{
FREE-ELECTRON LASERS: VACUUM ELECTRONIC GENERATORS OF COHERENT RADIATION
}

\author{
H.P. Freund \\ Science Applications International Corp. \\ McLean, VA 22102 \\ G.R. Neil \\ Thomas Jefferson National Accelerator Facility \\ 12000 Jefferson Avenue \\ Newport News, VA 23606
}

\begin{abstract}
Vacuum electronic sources have shown marked improvement since the invention of the magnetron before World War II, and dramatic increases in both average powers and frequencies have been achieved. Of course, much of these gains have been achieved by the development of different devices. The typical development pattern for a given device exhibits an initial period of rapid improvement followed by a plateau determined by technological or physical limitations on the concept. Slow wave devices such as magnetrons and/or klystrons operate efficiently at frequencies up through X-band or he source of the relation ed cavity traveling wave tubes are used for various applications at frequencies ranging up through W-band. At still higher frequencies fast wave devices such as gyrotrons and free-electron lasers are required are required for high power operation. The free-electron laser concept is unique in that the mechanism is applicable across the entire electromagnetic spectrum, and free-electron lasers have been built at wavelengths from microwaves through the ultraviolet, and plans are under development for X-ray systems. Our purpose of this paper is to describe the principal directions of free-electron laser research at the present time. To this end, we first give a brief tutorial of the physics underlying the concept, and then describe the principal development paths under way.
\end{abstract}




\section{Introduction}

The performance of vacuum electronic sources has shown marked improvement since the invention of the magnetron before World War II with dramatic increases in both average powers and frequencies. Of course, much of these gains have been achieved by the development of different devices. The typical development pattern for a given device exhibits an initial period of rapid improvement followed by a plateau determined by technological or physical limitations on the concept.

One figure of merit for the progress in vacuum electronic sources is the product of the average power and the square of the frequency which is a measure of the power density produced by the device. A plot showing the evolution of this figure of merit over time for the magnetron, klystron, gyrotron, and free-electron laser (FEL) is shown in Fig. 1 [1]. It is clear from the figure that rapid development of the magnetron occurred during and after World War II and reached its ultimate limits by about 1950. Klystron development followed a similar pattern over the period from the end of World War II through the mid-1970's. One important limitation faced by both of these devices stems from breakdown problems as the device sizes decrease with increases in frequency. This limitation can be partially overcome by the development of so-called fast-wave sources such as the gyrotron and FEL. In these devices, the beam interaction is mediated by an externally applied magnetic field and the phase velocities of the resonant waves can exceed the speed of light in vacuo. One practical benefit derived from this is that the circuits can be both simpler (i.e., smooth bore waveguides can be used) and larger; hence, the power handling capacity of the devices can be higher. In view of this, the development of the FEL and gyrotron progressed rapidly from the mid-1960's to the present.

The basic principles underlying the emission of radiation from electron beams propagating through periodic magnetic fields was first described by Hans Motz in 1951 [2-4]. At the time, coherent optical emission was not thought to be practical due the difficulty of bunching the beam at short wavelengths, although coherent radiation was 
produced at millimeter wavelengths. The concept was invented independently by Robert Phillips in 1957 when he realized that the periodic magnetic fields under development for beam focusing in traveling wave tubes also give rise to axial beam bunching. He coined the term ubitron as an acronym for Undulating Beam Interaction to describe these early freeelectron masers, and over the period 1957-1964 achieved powers as high as $150 \mathrm{~kW}$ at a wavelength of $5 \mathrm{~mm}$ and pioneered many innovative design concepts in use today $[5,6]$.

There are two principal directions in the future development of FELs. In the first place, one essential conclusion to be reached from Fig. 1 is that the envelope of the development curves for these devices has increased exponentially with $P_{\text {avg }} f^{2}$ increasing at the rate of almost two orders of magnitude per decade. At the present time, the question of whether development of the FEL has reached a plateau remains uncertain. One important direction for future research, therefore, is the quest for higher average powers at still higher frequencies. To this end, an FEL is under development at the Thomas Jefferson Accelerator Laboratory [7] whose goal is to reach an average power of $1 \mathrm{~kW}$ at a wavelength of 3 microns. As shown in the figure, this target is reasonable and consistent with the historical pace for vacuum electronic sources. In the second place, the resonant frequency in FELs increases with the square of the beam energy, and FELs have been demonstrated to operate over an unprecedented range of frequencies from microwaves through the ultraviolet [8]. The thrust here is to still shorter wavelengths; in particular, into the X-ray range and designs are under development for FELs with wavelengths as short as $1.5 \AA[9,10]$.

Our purpose in this paper is to provide an overview of these future developments. To that end, the organization of the paper is as follows. A brief description of the fundamental physics of the FEL is given in Sec. II, followed by a description of the major components of an FEL in Sec. III. The principal future directions of FEL development are described in Sec. IV, and a brief discussion of present and proposed applications is discussed in Sec. V. A summary is given in Sec. VI. 


\section{The Physics of Free-Electron Lasers}

The interaction between the electron beam and the output radiation field in an FEL is mediated by a periodic wiggler magnetic field. In conventional terminology, the periodic magnetic field in synchrotron light sources is referred to as an undulator while that used in FELs is called a wiggler, although there is no fundamental difference between them. As the electron beam traverses the wiggler field it emits incoherent radiation. It is necessary for the electron beam to form coherent bunches in order to give rise to the stimulated emission required for a free-electron laser. This can occur when a light wave traverses an undulatory magnetic field such as a wiggler because the spatial variations of the wiggler and the electromagnetic wave combine to produce a beat wave, which exerts a slowlyvarying ponderomotive force on the electrons. It is the interaction between the electrons and this beat wave which gives rise to stimulated emission. A schematic illustration of the process for a wiggler with planar symmetry is shown in Fig. 2

This ponderomotive wave has the same frequency as the light wave, but the wavenumber is the sum of the wavenumbers of the electromagnetic and wiggler fields. With the same frequency, but a larger wavenumber (and thus a shorter wavelength), the beat wave is subluminous and can be in synchronism with the electrons. Electrons moving in synchronism with the wave are said to be in resonance with it and will experience a constant field which can give rise to coherent amplification. Equating the phase velocity of the ponderomotive wave $\left[\mathrm{v}_{p h}=\omega /\left(k+k_{w}\right)\right.$, where $\omega$ and $k$ are the angular frequency and wavenumber of the wave and $k_{w}$ is the wiggler wavenumber] with the electron beam velocity, $\mathrm{v}_{b}$, we obtain the resonance condition

$$
\omega=\left(k+k_{w}\right) \mathbf{v}_{b}
$$

For waves propagating in free space $\omega=c k$ and this condition yields a wavelength of 


$$
\lambda=\frac{\lambda_{w}}{2 \gamma_{b}^{2}}
$$

where $\lambda_{w}$ is the wiggler period, and $\gamma_{b}=\left(1-\mathrm{v}_{b}{ }^{2} c^{2}\right)^{1 / 2}$. This is, of course, modified if the wavelength is comparable to either the radius of the drift tube walls or the beam plasma frequency.

In order to understand how a wiggler and a forward-propagating electromagnetic wave (whose electric and magnetic fields are oriented transversely to the electron beam) gives rise to an axial ponderomotive force which extracts energy from the electrons, we consider the particle motion. The wiggler is the predominant influence on the electron trajectories. In order to understand the dynamical relationships, consider electron motion in a helically symmetric wiggler. An electron propagating through the wiggler experiences a force which acts at right angles to both the direction of the field and to its own velocity. The wiggler field is directed transversely to the direction of bulk motion and rotates through $360^{\circ}$ in one wiggler period. A streaming electron, therefore, experiences a transverse force and acquires a transverse velocity component upon entry into the wiggler. The resulting orbit is helical and describes bulk streaming along the symmetry axis as well as a transverse circular rotation that lags $180^{\circ}$ behind the phase of the wiggler. The magnitude of the transverse wiggle velocity, denoted by $\mathrm{v}_{w}$, is proportional to the product of the wiggler amplitude and period. This relationship may be expressed in the form

$$
\frac{\mathrm{v}_{w}}{c}=\frac{a_{w}}{\gamma_{0}}=0.934 \frac{B_{w} \lambda_{w}}{\gamma_{0}}
$$

where $a_{w}\left[+e B_{w} / m_{e} c^{2} k_{w}^{2}\right]$ is the wiggler strength parameter, the wiggler period is expressed in units of centimeters, $B_{w}$ denotes the wiggler amplitude in Tesla, and $\gamma_{0}=1+$ $E_{b} / m_{e} c^{2}$ denotes the relativistic time dilation factor associated with the total kinetic energy $E_{b}$ of the electron beam (where $m_{e}$ denotes the rest mass of the electron, and $m_{e} c^{2}$ denotes the electron rest energy). Since the motion is circular, both axial and transverse velocities 
have a constant magnitude, and the relation between the total electron energy and the streaming energy can be expressed as

$$
\gamma_{b}=\frac{\gamma_{0}}{\sqrt{1+a_{w}^{2}}}=\frac{\gamma_{0}}{\sqrt{1+0.872 B_{w}^{2} \lambda_{w}^{2}}}
$$

As a result, the resonant wavelength depends upon the total beam energy, and the wiggler amplitude and period through

$$
\lambda=\left(1+a_{w}^{2}\right) \frac{\lambda_{w}}{2 \gamma_{0}^{2}}=\left(1+0.872 B_{w}^{2} \lambda_{w}^{2}\right) \frac{\lambda_{w}}{2 \gamma_{0}^{2}} .
$$

It is the interaction between the transverse wiggler-induced velocity with the transverse magnetic field of an electromagnetic wave that induces the ponderomotive force normal to both in the axial direction. The transverse velocity and the radiation magnetic field are directed at right angles to each other and undergo a simple rotation about the axis of symmetry. A resonant wave must be circularly polarized with a polarization vector that is normal to both the transverse velocity and the wiggler field and which rotates in synchronism with the electrons. This synchronism is illustrated in Fig. 3 [8], and is maintained by the aforementioned resonance condition.

The interaction in a wiggler with planar symmetry is qualitatively similar; however, there are some significant differences. In a planar wiggler, the transverse motion is directed linearly; hence, the oscillatory component is not of constant amplitude. This, in turn, introduces an oscillatory component to the axial velocity. Because of this, the wiggler field magnitude used in the formulae obtained for a helical wiggler must be replaced by the rms magnitude when applied to a planar wiggler. As such, a planar wiggler field must be approximately v2 times stronger than a helical wiggler field to have a comparable effect.

\section{A. Spontaneous Emission}


The incoherent spontaneous emission of a beam in a wiggler has been discussed by several authors $[4,8,11-13]$ and can be expressed in terms of the emissivity

$$
\eta\left(\omega, \Omega_{\mathbf{k}}\right) \equiv \frac{1}{V} \frac{d P}{d \omega d \Omega_{\mathbf{k}}}
$$

that describes the power emitted per unit volume $V$, per unit frequency $\omega$, per unit solid angle $\Omega_{\mathbf{k}}$ along the direction of the wavevector $\mathbf{k}$. In the cold beam regime where $\Delta \mathrm{v}_{b} / \mathrm{v}_{b}<$ $\lambda / L$ (where $L$ is the length of the wiggler), the emissivity for a helical wiggler is azimuthally symmetric and takes the form

$$
\begin{aligned}
\eta(\omega, \theta)=\frac{\omega^{2} L}{(2 \pi)^{3}} \frac{\omega_{b}^{2} m_{e} a_{w}^{2}}{2 \gamma_{0}^{2} c \mathbf{V}_{b}} \sum_{n=-\infty}^{\infty}\left[\left(\cos \theta-\frac{k}{n k_{w}} \sin ^{2} \theta\right)^{2} \frac{n^{2}}{b^{2}} J_{n}^{2}(b)+J_{n}{ }^{\prime 2}(b)\right] \\
\times\left(\frac{\sin \Theta_{n}}{\Theta_{n}}\right)^{2}
\end{aligned}
$$

where $\omega_{b}^{2}+4 \pi \mathrm{e}^{2} n_{b} / m_{e}$ is the square of the beam plasma frequency for an ambient beam density of $n_{b}, a_{w} \sim e B_{w} / m_{e} c^{2} k_{w}$ is the wiggler strength parameter, $\theta=\tan ^{-1}\left(k_{\perp} / k_{\|}\right)$is the polar angle between the wavevector and the symmetry axis, $J_{n}$ is the regular Bessel function of the first kind of order $n, b=k_{\perp} \mathrm{v}_{w} / k_{w} \mathrm{v}_{b}$, and

$$
\Theta_{n}=\frac{L}{2 \mathrm{v}_{b}}\left[\omega-\left(k+n k_{w}\right) \mathrm{v}_{b}\right]
$$

The emission band at each harmonic is determined by the spectral function $\sin ^{2} \Theta_{n} / \Theta_{n}{ }^{2}$ which is plotted in Fig. 4. The spontaneous emission spectrum peaks for a zero frequency mismatch [i.e., $\Theta_{n}=0$ ]; hence

$$
\omega=\frac{n k_{w} \mathbf{V}_{b}}{1-\left(\mathrm{V}_{b} / c\right) \cos \theta}
$$

which becomes $\omega^{\sim} 2 \gamma_{b}^{2} k_{w} \mathrm{v}_{b}$ in the ultra-relativistic limit. The FWHM spectral width is determined by the central peak which yields $-1.4=\Theta_{n}=1.4$; consequently,

$$
\frac{\Delta \omega}{\omega}<\frac{\lambda_{w}}{L}
$$


The emission peaks in the forward direction [i.e., $\theta=0$ ]; hence, we may take the limit $b<<1$ to obtain

$$
\eta(\omega, \theta) \cong \frac{\omega^{2} L}{(2 \pi)^{3}} \frac{\omega_{b}^{2} m_{e} a_{w}^{2}}{8 \gamma_{0}^{2} c \mathrm{v}_{b}}\left[1+\left(\cos \theta-\frac{k}{n k_{w}} \sin ^{2} \theta\right)^{2}\right]\left(\frac{\sin \Theta_{n}}{\Theta_{n}}\right)^{2}
$$

This yields an on-axis peak emissivity of

$$
\eta(\omega, \theta=0) \cong \frac{\omega^{2} L}{(2 \pi)^{3}} \frac{\omega_{b}^{2} m_{e} a_{w}^{2}}{4 \gamma_{0}^{2} c \mathrm{~V}_{b}}\left(\frac{\sin \Theta_{1}}{\Theta_{1}}\right)^{2}
$$

and an angular spread in which the polar angle at the FWHM point is

$$
\theta_{F W H M} \cong 1.3 \sqrt{\frac{\lambda}{L}}
$$

Emission at the harmonics is more sharply peaked.

The spontaneous emission from a planar wiggler system is similar, but differs in that the simpler symmetry properties break the azimuthal symmetry exhibited by the helical wiggler. This is discussed in ref. [14].

\section{B. Coherent Amplification}

FELs can be configured in three general configurations: oscillators, Master Oscillator Power Amplifiers (MOPAs), and Self-Amplified Spontaneous Emission (SASE) amplifiers. The signal in MOPA amplifiers grows from a seed signal injected from an external source termed the master oscillator; hence, this configuration requires an additional source of coherent radiation. In contrast, the spontaneous emission is used as the source in oscillators and SASE amplifiers. In oscillators, the radiation is confined to a cavity in which only a small fraction of the power leaves the cavity on each pass through the wiggler. In such systems, the radiation gain per pass is very small (typically only a few percent), and the transverse mode structure is governed predominantly by the cavity geometry. Oscillators are useful at wavelengths for which good mirrors exist and for 
systems in which the electron beam currents are too low to obtain high gains per pass through the wiggler. One variation on this design is referred to as a regenerative amplifier [15]. A cavity is also used in a regenerative amplifier, but the gain per pass of the radiation through the wiggler is large and a large fraction of the signal leaves the cavity after each pass through the wiggler. As a result, the signal grows from noise [i.e., from the spontaneous emission] to saturation in only a few passes through the wiggler, and the transverse mode structure is less dependent upon the cavity geometry. A SASE amplifier is one in which the gain per pass through the wiggler is large enough that the radiation grows from noise to saturation in a single pass through the wiggler. This requires either intense electron beams or long/high amplitude wigglers to obtain high enough growth rates; however, it has the advantage that no mirrors are required for ultra-short wavelength operation.

Amplification is described by a nonlinear pendulum equation. The ponderomotive phase $\psi\left[=\left(k+k_{w}\right) z-\omega t\right]$ is a measure of the position of an electron in both space and time with respect to the ponderomotive wave. In order to understand this consider the motion of an electron in a combined helical wiggler (in the one-dimensional limit)

$$
\mathbf{B}_{w}=B_{w}\left[\hat{\mathbf{e}}_{x} \cos k_{w} z+\hat{\mathbf{e}}_{y} \sin k_{w} z\right]
$$

and a circularly polarized electromagnetic wave given by the vector potential

$$
\delta \mathbf{A}=\delta A\left[\hat{\mathbf{e}}_{x} \cos (k z-\omega t)-\hat{\mathbf{e}}_{y} \sin (k z-\omega t)\right]
$$

The Lorentz force equations in the wiggler frame are given by

$$
\frac{d}{d t} \mathbf{v}=\frac{e}{\gamma m_{e}^{c}}\left(\mathbf{I}-\frac{1}{c^{2}} \mathbf{v v}\right) \cdot \frac{\partial}{\partial t} \delta \mathbf{A}-\frac{e}{\gamma m_{e} c} \mathbf{v} \times\left[\mathbf{B}_{w}+\nabla \times \delta \mathbf{A}\right],
$$

$$
\frac{d}{d t} \gamma=\frac{e}{m_{e} c^{3}} \mathbf{v} \cdot \frac{\partial}{\partial t} \delta \mathbf{A}
$$


where $\mathbf{I}$ is the unit dyadic. Solutions to these equations are obtained by perturbation to first order in the radiation field of the form $\mathbf{v}=\mathbf{v}_{0}+\delta \mathbf{v}$ and $\gamma=\gamma_{0}+\delta \gamma$. To lowest order, Eq. (16) becomes

$$
\frac{d}{d t} \mathbf{v}_{0}=-\frac{e}{\gamma_{0} e_{e}} \mathbf{v}_{0} \times \mathbf{B}_{w}
$$

This equation is characterized by steady-state helical trajectories

$$
\mathbf{v}_{0}=-\frac{a_{w}}{\gamma_{0}}\left[\hat{\mathbf{e}}_{x} \cos k_{w} z+\hat{\mathbf{e}}_{y} \sin k_{w} z\right]+\mathbf{v}_{b} \hat{\mathbf{e}}_{z}
$$

where

$$
\frac{\mathrm{v}_{b}}{c}=\sqrt{1-\frac{1+a_{w}^{2}}{\gamma_{0}^{2}}}
$$

To first order we have

$$
\frac{d}{d t} \delta \mathbf{v}=-\frac{e}{\gamma m_{e} c^{c}} \delta \mathbf{v} \times \mathbf{B}_{w}+\frac{e}{\gamma_{0} m_{e} c}\left[\left(\mathbf{I}-\frac{1}{c^{2}} \mathbf{v}_{0} \mathbf{v}_{0}\right) \cdot \frac{\partial}{\partial t} \delta \mathbf{A}-\mathbf{v}_{0} \times(\nabla \times \delta \mathbf{A})\right]
$$

The axial component of this equation

$$
\frac{d}{d t} \delta \mathrm{v}_{3}=-2 k_{w} c^{2} \frac{a_{w} \delta a}{\gamma_{0}^{2}} \sin \psi
$$

derives from the $\mathbf{v}_{0} \times(\nabla \times \delta \mathbf{A})$ term [which is proportional to $\mathbf{B}_{w} \infty \delta \mathbf{B}$ ], where $\delta a=$ $e \delta A / m_{e} c^{2}$. This describes the ponderomotive force which gives rise to axial bunching of the electron beam. Noting that $\delta v_{3} \approx v_{b}^{3} \psi^{\prime \prime} / \omega$ from the definition of the ponderomotive phase (where the ' denotes a derivative with respect to $z$ ), we obtain the pendulum equation

$$
\frac{d^{2}}{d z^{2}} \psi=-\kappa_{p o n d}^{2} \sin \psi
$$

where

$$
\kappa_{\text {pond }}=2 k_{w} \sqrt{\frac{a_{w} \delta a}{1+a_{w}^{2}}},
$$


is the pendulum constant in the relativistic limit where $\mathrm{v}_{b} \sim c$. This applies for a planar wiggler as well subject to the substitution of the rms wiggler amplitude in $a_{w}$. There are two classes of trajectory for the pendulum equation: trapped and untrapped. The electrons on untrapped trajectories pass over the crests of many waves traveling fastest at the bottom of the troughs and slowest at the crests, while the electrons are confined within the troughs in the trapped orbits. The separatrix between the trapped and untrapped regimes is given by

$$
\frac{d}{d z} \psi= \pm 2 \kappa_{\text {pond }} \cos \left(\frac{\psi}{2}\right) \text {. }
$$

The dynamical evolution of the electron phase space during the coherent emission process is illustrated schematically in Fig. 5. If the electrons are initially monoenergetic, then the phase space distribution is a horizontal line as illustrated in Fig. 5a. Note that the pendulum constant evolves during the course of the interaction as the wave amplitude grows. Since amplification has only just begun, the wave is of small amplitude and the separatrix encloses a small area of phase space. The electrons lose energy as the wave is amplified and decelerate [i.e., $d \psi / d z$ decreases] while both the pendulum constant and separatrix grow. During the linear phase of the interaction (Fig. 5b) the electrons have only begun to form bunches and remain on untrapped trajectories. Note also that the bulk $d \psi / d z$ for the electron distribution has dropped since the electrons have lost energy in the linear phase of the interaction. Ultimately, the electrons cross the growing separatrix onto trapped orbits, and the interaction saturates after the electrons have executed approximately half of an oscillation in the ponderomotive well. At this point, the electrons which are still losing energy to the wave are balanced by those electrons which are gaining energy at the expense of the wave.

In general, the dynamic evolution of electromagnetic waves in an FEL is a multistage amplification process with three distinct phases as indicated schematically in Fig. 6. The first phase is the low gain regime over a short initial distance from the start of the 
wiggler during which the wave grows roughly as the cube of the distance after which the second, exponential growth phase occurs. The exponentiation length is defined as the length over which the wave amplitude experiences one e-folding during this second phase of the interaction. Typically, the wiggler must be longer than several exponentiation lengths in order for the system to progress beyond the low gain regime and reach the exponential phase. The third, and nonlinear, phase of the interaction begins once the power has reached a level at which electrons start crossing the separatrix from untrapped to trapped orbits.

The interaction proceeds somewhat differently for low-gain FEL oscillators employing either short wigglers or low current electron beams. In such devices, the exponentiation length is too long for the interaction to progress beyond the low gain regime over the length of the wiggler. As such, the wave amplitude grows only by a small amount on each pass through the wiggler, and the exponentiation phase never takes place for a given electron bunch. However, the radiation amplitude grows to levels able to trap the electron beam over many passes through the wiggler, and the saturation mechanism is the same phase trapping process described in Fig. 5 and which is operable when exponential growth occurs.

In describing this linear phase of the interaction, we first consider the initial lowgain regime. Since this process is applicable largely to low-gain oscillators employing low current electron beams, we limit the discussion to the Compton regime. In this regime, beam space-charge effects are negligible, and the interaction can be thought of as coherent Compton scattering of the wiggler (which appears to be an electromagnetic wave in the electron rest frame) off of the electron beam. For a cold electron beam, the power in the early stages of the interaction grows as the cube of the distance and the gain can be expressed as [3 and references contained therein] 


$$
G(L)=\sigma_{A} \frac{\omega_{b}^{2} a_{w}^{2} L^{3} c}{2 \gamma_{0}^{3} \mathrm{v}_{b}^{3}} F\left(\Theta_{1}\right)
$$

where $\sigma_{A}$ denotes the filling factor which describes the relative overlap of the electron beam and the radiation field, and

$$
F(x)=\frac{d}{d x}\left(\frac{\sin x}{x}\right)^{2}
$$

denotes the gain spectral function. Observe that in this regime, the gain is proportional to the derivative of the spontaneous emission with respect to $\Theta$. This property of the gain was proven rigorously in Madey's Theorem [16] The spectral function is shown in Fig. 7, and exhibits the extrema $F( \pm 1.3) \sim \mathrm{m} 0.54$. Hence, the maximum gain is found at a frequency which is detuned from the exact resonance and is given by

$$
\omega \cong \frac{k_{w} \mathrm{v}_{b}}{1-\mathrm{v}_{b} / c}\left(1-\frac{2.6}{k_{w} L}\right)
$$

As in the case of the spontaneous emission, thermal effects become important when $\Delta \mathrm{v}_{b} / \mathrm{v}_{b}$ $<\lambda / L$. This gain result is modified for a planar wiggler to account for the effect of the oscillating axial velocity. In this case, the gain at the odd harmonics is governed by

(29) where $m$ is an integer,

$$
G(L)=\sigma_{A} \frac{\omega_{b}^{2} a_{w}^{2} L^{3} c}{4 \gamma_{0}^{3} \mathbf{v}_{b}^{3}} K_{m}\left(b_{m}\right) F\left(\Theta_{2 m+1}\right),
$$

and

$$
K_{m}\left(b_{m}\right) \equiv\left[J_{m}\left(b_{m}\right)-J_{m \pm 1}\left(b_{m}\right)\right]^{2}
$$

$$
b_{m}=(2 m+1) \frac{a_{w}^{2} / 2}{1+a_{w}^{2} / 2} \text {. }
$$

The low gain regime described above is relevant to situations where the total gain over the length of the system is much less than unity. A dispersion equation is solved in the exponential gain regime in order to determine the growth rate which, in the one- 
dimensional limit for a helical wiggler and a cold electron beam, is of the form [3 and references contained therein]

$$
\left[\left[\omega-\left(k+k_{w}\right) \mathrm{v}_{b}\right]^{2}-\frac{\omega_{b}^{2}}{\gamma_{0} \gamma_{b}^{2}}\right]\left(k-k_{0}\right) \cong-\frac{\omega_{b}^{2} a_{w}^{2} k_{w}}{2 \gamma_{0}^{3}}
$$

where $k_{0}=\left(\omega^{2}-\omega_{b}^{2} / \gamma_{0}\right)^{1 / 2} / c$ describes the wavenumber of the electromagnetic wave. This dispersion equation describes the coupling of the electromagnetic wave $\left(k \sim k_{0}\right)$ with positive and negative energy space-charge waves $\left[\omega-\left(k+k_{w}\right) \mathrm{v}_{b}= \pm \omega_{b} / \gamma_{0}{ }^{1 / 2} \gamma_{b}\right]$. The high-gain Compton regime is found in the limit

$$
\frac{\omega_{b}}{\gamma_{0}^{1 / 2} k_{w} c}<<\frac{\gamma_{b}}{16} \frac{a_{w}^{2}}{1+a_{w}^{2}}
$$

where the space-charge waves can be neglected. In this limit, the cubic dispersion equation exhibits a maximum growth rate at zero detuning [i.e., $\left.\omega^{\sim} k_{w} \mathrm{v}_{b} /\left(1-\mathrm{v}_{b} / c\right)\right]$ of

$$
\frac{\operatorname{Im} k}{k_{w}}=\frac{\sqrt{3}}{2 \gamma_{0}}\left(\frac{a_{w}^{2}}{2} \frac{\omega_{b}^{2}}{c^{2} k_{w}^{2}}\right)^{1 / 3}
$$

The opposite limit where the space-charge waves are dominant is referred to as the collective Raman regime. Here the interaction is stimulated scattering of the negative energy space-charge wave and the wiggler to produce the output (daughter) wave, and a maximum growth rate of

$$
\frac{\operatorname{Im} k}{k_{w}}=\frac{a_{w}}{2 \gamma_{0}}\left(\frac{\gamma_{b} \omega_{b}}{\gamma_{0}^{1 / 2} c k_{w}}\right)^{1 / 2}
$$

is found for $\omega=\left(k_{0}+k_{w}\right) \mathrm{v}_{b}-\omega_{b} / \gamma_{0}^{1 / 2} \gamma_{b}$.

Thermal effects become important in the exponential gain regime when $\Delta \mathrm{v}_{b} / \mathrm{v}_{b} \sim$ $\operatorname{Im} k /\left(\operatorname{Re} k+k_{w}\right)$. This implies that thermal effects cannot be ignored when 


$$
\frac{\Delta \mathrm{v}_{b}}{\mathrm{v}_{b}} \approx \frac{\sqrt{3}}{4 \gamma_{b}^{2}}\left(\frac{a_{w}^{2}}{2} \frac{\omega_{b}^{2}}{\gamma_{0} k_{w}^{2} c^{2}}\right)^{1 / 3}
$$

in the Compton regime, and

$$
\frac{\Delta \mathrm{v}_{b}}{\mathrm{v}_{b}} \approx \frac{a_{w}}{4 \gamma_{b}^{2}}\left(\frac{\omega_{b}}{\gamma_{0}^{1 / 2} k_{w} c}\right)^{1 / 2}
$$

in the Raman regime. However, if an FEL is operating in the thermal regime, then the gain and efficiency are too low to make a practical device. Because of this, a great deal of effort is expended to generate high quality electron beams.

An important distinction between the low gain and exponential gain regimes is the tuning of the interaction at peak gain. In the low gain regime, the maximum gain is shifted away from the exact wave-particle resonance [Eq. (29)], while the maximum growth rates in the exponential gain regime are found for $\omega^{\sim} k_{w} \mathrm{v}_{b} /\left(1-\mathrm{v}_{b} / c\right)$ in the limit in which $\omega_{b}<<$ $\gamma_{0}{ }^{1 / 2} \gamma_{b} k_{w} \mathrm{v}_{b}$

The corresponding dispersion equation for planar wigglers is obtained in a similar way as described for the low gain regime to handle the oscillatory axial velocity. That is, the dispersion equation now takes the form [3 and references contained therein]

$$
\left[\left[\omega-\left(k+k_{w}\right) \mathbf{v}_{b}\right]^{2}-\frac{\omega_{b}^{2}}{\gamma_{0} \gamma_{b}^{2}}\right]\left(k-k_{0}\right) \cong-\frac{\omega_{b}^{2} a_{w}^{2} k_{w}}{4 \gamma_{0}^{3}} K_{m}\left(b_{m}\right)
$$

that is similar to Eq. (32) and differs only in the substitution of the rms value of $a_{w}$ and the inclusion of the $K_{m}$ factor. Hence, the results found for the helical wiggler geometry carry over to the planar wiggler geometry by the substitution $a_{w} \varnothing a_{w} K_{m}\left(b_{m}\right) / 2$.

Further generalizations of the growth rates in the exponential gain regime to threedimensions are too complicated to present within the context of the present work. However, three-dimensional analyses have been published for helical [17] and planar $[18,19]$ wiggler geometries. 
As mentioned previously, the low gain regime represents the initial stages of the interaction prior to the onset of exponentiation that occurs after, at most, several exponentiation lengths. In practice, this means that the low gain regime holds only when the system is shorter than an exponentiation length. Hence, the low gain regime is applicable only when

$$
L<<\frac{\gamma_{0} \lambda_{w}}{2 \pi} \begin{cases}\frac{1}{\sqrt{3}}\left(\frac{a_{w}^{2}}{2} \frac{\omega_{b}^{2}}{c^{2} k_{w}^{2}}\right)^{-1 / 3} & ; \text { Compton regime } \\ \left(\frac{\gamma_{b} a_{w}^{2} \omega_{b}}{\gamma_{0}^{1 / 2} c k_{w}}\right)^{-1 / 2} & ; \text { Raman regime }\end{cases}
$$

The saturation efficiency can be estimated from the requirement that the net change in electron velocity at saturation is equal to twice the velocity difference between the electron beam and the ponderomotive wave. This technique dates back to J.C. Slater [20] in 1950 who applied it to traveling wave tubes; however, the technique works equally well for FELs. In the low gain regime, this phase trapping criterion results in an efficiency, $\eta$, of

$$
\eta \approx \frac{\lambda_{w}}{2 L}
$$

while in the high-gain Compton and collective Raman regimes we obtain

$$
\eta \approx \frac{1}{2 \gamma_{0}}\left(\frac{a_{w}^{2}}{2} \frac{\omega_{b}^{2}}{\gamma_{0} k_{w}^{2} c^{2}}\right)^{1 / 3}
$$

and

$$
\eta \approx \frac{\omega_{b}}{\gamma_{0}^{1 / 2} \gamma_{b} k_{w} c}
$$

respectively. These "crude" estimates give reliable estimates of the efficiency of an FEL with a uniform wiggler field. 
In the post-saturation regime, the electron motion in the ponderomotive potential results in the growth of sidebands. This issue is not greatly important in MOPA and SASE amplifiers since the wiggler length can be adjusted to minimize this phase of the interaction. However, sidebands can play an important role in FEL oscillators where they compete with, and drain energy from, the fundamental. Sideband control, therefore, is an important issue in oscillator design. An extensive discussion of sideband growth and control is also beyond the scope of this paper. However, we note that sidebands have been effectively controlled by the use of tapered wigglers [21,22], and Littrow gratings [23].

\section{Tapered Wigglers}

The efficiency can be enhanced by tapering the amplitude and/or period of the wiggler. A relatively simple physical explanation for this effect is found by noting that as the electrons lose energy to the wave they decelerate and drop out of resonance. Hence, more energy could be extracted if the electrons are re-accelerated. Since the transverse wiggler-induced velocity is proportional to the product of the wiggler amplitude and period, a tapered reduction in either or both of these parameters results in a decrease in the transverse electron velocity and a corresponding increase in the axial velocity. This procedure requires that the taper begin at a point prior to saturation but after the beam has crossed the separatrix onto trapped trajectories. The pendulum equation governing particle dynamics in a tapered wiggler is of the form

$$
\frac{d^{2}}{d z^{2}} \psi=-\kappa_{\text {pond }}^{2}\left(\sin \psi-\sin \psi_{\text {res }}\right)
$$

where 


$$
\sin \psi_{\text {res }}=-\frac{k_{w}}{\kappa_{\text {pond }}^{2}}\left[\frac{\lambda_{w}^{\prime}}{\lambda_{w}}+\frac{a_{w}^{2}}{1+a_{w}^{2}}\left(\frac{B_{w}^{\prime}}{B_{w}}+\frac{\lambda_{w}^{\prime}}{\lambda_{w}}\right)\right]
$$

and the prime (') superscript denotes a derivative with respect to $z$. This reduces to Eq. (23) for a uniform wiggler in the limit in which the derivatives of the wiggler amplitude and period vanish.

Once the beam has become trapped, the axial acceleration due to the wiggler taper results in an increase in the efficiency of [8]

$$
\Delta \eta=-\frac{\Delta \lambda_{w}}{2 \lambda_{w}}-\frac{a_{w}^{2}}{1+a_{w}^{2}}\left(\frac{\Delta B_{w}}{B_{w}}+\frac{\Delta \lambda_{w}}{\lambda_{w}}\right)
$$

In the case where the period is held fixed and the amplitude is tapered, this implies that the theoretical maximum increase in the efficiency is $\Delta \eta \sim a_{w}{ }^{2} /\left(1+a_{w}{ }^{2}\right)$. The case where the period is tapered is more complicated. The periodic variation of the field in a wiggler results from the partial cancellation of the field contributions from adjacent (and oppositely directed) magnets. Hence, as the separation between adjacent magnets (i.e., the wiggler period) decreases, the wiggler amplitude decreases as well.

However, this theoretical maximum is seldom achieved in practice. One reason for this is that the underlying assumption in the derivation of (45) that the entire beam has been trapped by the ponderomotive wave is an idealization. In practice, the maximum realizable efficiency enhancement must be reduced by the trapping fraction, i.e., the proportion of the beam which is actually trapped by the ponderomotive wave. It is important to recognize in this regard that the trapping fraction is very sensitive to the beam emittance. The axial energy spread increases with the emittance, and must be smaller than the depth of the ponderomotive wave in order for a large fraction of the beam to be trapped. As a result, the maximum achievable efficiency enhancement decrease with increases in the emittance. Another significant limitation is that the gain length increases 
and bucket size decreases as the magnetic field is reduced thus leading to practical limits on how far the taper can be extended.

\section{Optical Guiding}

Optical guiding refers to the self-focusing of the electromagnetic wave by the electron beam $[8,24-26]$. Optical guiding occurs by two related mechanisms referred as gain and refractive guiding. Gain guiding describes the preferential amplification of radiation in the region occupied by the electron beam. Refractive guiding describes the focusing (or defocusing) of the radiation by means of the shift in the refractive index due to the dielectric response of the electron beam. In particular, if the wavenumber is shifted upward due to the interaction with respect to the vacuum state, then the phase velocity of the wave decreases and the beam acts as an optical waveguide. It should be remarked, however, that gain and refractive guiding are intimately linked and are not independent processes.

Optical guiding is not an important effect in free-electron lasers operating in the microwave regime where the drift tube acts as a waveguide; however, even in this regime the shift in the wavenumber results in a variation in the phase of the amplified signal relative to the vacuum propagation of the wave which can be measured. In contrast, optical guiding is an important effect in the short wavelength regime. Here, free-space diffraction would cause the optical field to expand away from the electron beam over a scale length of the order of a Rayleigh range, and would significantly degrade the interaction. In practice, however, optical guiding permits the co-propagation of the electromagnetic wave with the electron beam over the course of many Rayleigh lengths.

\section{E. Slippage and Lethargy}

In most systems, the group velocity of the radiation is higher than the axial velocity of the electron beam and this results in the slippage of the beam behind the radiation. One exception to this rule is possible for long wavelength FELs where the dispersion of the 
radiation is governed by the waveguide geometry where the group velocity is given by $\mathrm{v}_{g r}$ $=c\left(1-\omega_{c o}{ }^{2} / \omega^{2}\right)^{1 / 2}$, where $\omega_{c o}$ denotes the cutoff frequency of the specific waveguide mode of interest. Since the cutoff frequency is determined by the waveguide dimensions, it is possible to control the group velocity, and this technique was used at Columbia University [21,22] and at Frascati [27] to detune the sidebands in an FEL oscillator. However, we are primarily concerned in this paper with short wavelength FELs where the frequencies are much greater than either the beam plasma frequency or any cutoff frequencies of the drift tube and $\mathrm{v}_{g r} \sim c$. In this regard, the effect of slippage is somewhat different in FEL oscillators and amplifiers and we will discuss these two cases separately.

Slippage is not an important consideration in amplifiers driven by a continuous electron beam; however, radiation growth from a drive signal (MOPA) or noise (SASE) in a single pass through the wiggler can be adversely affected by slippage in a pulsed electron beam system. If we use $T_{w}=L / \mathrm{v}_{g r} \sim L / c$ to denote the transit time of the radiation through a wiggler of length $L$, then the slippage distance of an electron pulse relative to the radiation is $s=L\left(1-\mathrm{v}_{b} / \mathrm{v}_{g r}\right) \sim L\left(1-\mathrm{v}_{b} / c\right) \sim L / 2 \gamma_{b} \sim N_{w} \lambda$ for $\mathrm{v}_{g r} \sim c$ and $\mathrm{v}_{b} \sim c$ where $N_{w}$ denotes the number of periods in the wiggler. If $\tau_{p}$ denotes the duration of the electron beam pulse, then slippage can be neglected if the slippage time $\tau_{s}\left(+s / \mathrm{v}_{b}\right) \ll \tau_{p}$ which implies that

$$
\lambda<<\lambda_{w} \frac{\tau_{p}}{T_{w}} .
$$

As an example, we consider the proposed Linac Coherent Light Source (LCLS) at SLAC [9] in which a $15 \mathrm{GeV} / 5 \mathrm{kA}$ electron beam with pulse durations of the order of 8-10 psec generates X-rays at wavelengths of about $1.5 \AA$ using a $55 \mathrm{~m}$ long wiggler with a period of $3 \mathrm{~cm}$. For this proposed application, the slippage time $\tau_{s} \sim 8 \infty 10^{-15} \mathrm{sec}$ is three orders of magnitude less than the pulse duration, and (46) is well-satisfied. Hence, slippage will have a negligible effect on the proposed LCLS. 
Lethargy is a term used to describe distortions of the output optical pulse shape related to the slippage of the beam relative to the optical signal. Consider the case of a MOPA amplifier in which a drive pulse is injected in synchronism with the electron beam pulse. In the first place, the leading edge of the optical pulse will be amplified only for a short time before it slips ahead of the electron beam, after which it will decay due to wall losses and diffraction. The trailing edge of the pulse, however, will experience amplification over a longer period of time. In the second place, the interaction with the electron beam causes a refractive slowing of the optical pulse. This magnifies the effect of the slippage since once the leading edge of the optical pulse slips ahead of the electron pulse it races further ahead since the group velocity is locally larger than the group velocity within the electron beam. In the third place, there is also some delay associated with the response of the electrons to the optical pulse. This is similar to the previous discussion of the low gain regime which precedes the start of the exponential growth phase of the interaction, and derives from the fact that the electrons require some period in which develop some phase coherence (i.e., bunching) before growth can begin in earnest. As a result, the leading edge of the optical pulse starts the bunching process before it slips ahead of the beam, but it is the trailing edge of the optical pulse which benefits the greatest. As a result of these three effects, the optical pulse shape upon exiting from the wiggler will have a distorted shape in which the trailing edge has undergone more amplification than the leading edge.

Slippage and lethargy are also important considerations in an oscillator driven by a pulsed electron beam, and it is important to ensure that the slippage time is less than the pulse duration for oscillators. One exception to this rule is found when the cavity losses per pass are extremely small compared to the gain. In this limit, operation is possible because there is enough gain per pass to compensate for the desynchronism between the electromagnetic field and the electron beam [28] In oscillators, however, the effect of lethargy is to modify the cavity detuning. In order for there to be synchronism between the 
electron pulses and the optical pulses in an oscillator, the separation time between beam pulses must equal the round trip time of the optical pulses in the cavity. This is referred to as the cavity tuning. As a result, because of the refractive slowing of the optical pulse by the beam the cavity length must be shorter than would be indicated by the round trip time in the cavity in vacuo.

\section{F. Quantum Mechanical Effects}

In general quantum mechanical effects can be neglected when the spreading of the electron wave-packet is less than one wave period over the length of the wiggler $L$. This spreading, $\Delta z$, can be shown to be small and quantum mechanical effects can be neglected when $[8,29,30]$

$$
\Delta z=\frac{\lambda_{c} L}{\gamma_{0} \lambda_{w}}<\lambda,
$$

where $\lambda_{c}+h / m_{e} c$ is the Compton wavelength. This is well-satisfied for virtually all cases of practical interest. For example, consider a $10.6 \mu \mathrm{m}$ wavelength FEL which employs a $20 \mathrm{MeV}$ electron beam and a wiggler which is 100 periods in length. In this case, $\Delta \mathrm{z}^{\sim} 6 \infty$ $10^{-6} \mu \mathrm{m}$ and the inequality is satisfied by a more than six orders of magnitude. Quantum mechanical effects can also be neglected in the case of the LCLS where $\Delta z^{\sim} 9.2 \infty 10^{-4}$ $\AA$, which is still three orders of magnitude less than the $1.5 \AA$ wavelength.

Another requirement for the neglect of quantum mechanical effects is that the electron recoil upon the emission of a photon be small. This criterion may be stated in the form that the downshift in the frequency of the emitted photon due to the electron recoil must be much smaller than the gain linewidth. However, this requirement results in a condition which is identical to (47).

\section{The Principal Components of Free-Electron Lasers}


Like other lasers, the FEL consists of a gain medium, a means to put energy into it, a means for dealing with the spent energy, and an optical system to appropriately direct the photons produced. In an amplifier mode, it must also include an initial source of radiation. The gain medium in the FEL is the electron beam produced various types of accelerator. Electron accelerators are a relatively well-developed technology and the engineering involved is well known; however, the FEL puts extreme demands on the quality of the electron beam and care must be taken of the details of accelerator design. Indeed, the feasibility of FEL designs have always hinged on the beam brightness produced by the accelerator. The output of the FEL mimics to a great extent the temporal characteristics of the electron source so that the desired radiation characteristics influence the choice of accelerator technology.

A schematic of a typical RF Linac-driven FEL is shown in Fig. 8, although the figure shows a photocathode electron gun and a superconducting RF Linac. The principal FEL subsystems are common to all such: accelerator, injector, wiggler and focusing magnets, and optical systems. Note that an FEL amplifier or SASE configuration would be similar, but would omit the optical system. We shall discuss each of these subsystems in turn in the remainder of this section.

\section{A. Accelerators}

At wavelengths ranging from millimeter waves to the mid-infrared, DC accelerators such as pulse line accelerators and modulators can be used to accelerate the beam from a either a thermionic or field emission cathode. Induction linacs have been used at these wavelengths as well. At these longer wavelengths, the FEL gain can be very high and very high peak powers can be produced. DC sources also have the potential of producing high average power at high efficiencies if some means of recovering the beam energy or current is used. 
In the case of modulators and pulse line accelerators, energy recovery is accomplished by means of depressed collectors, and the spent beam is not recycled. At the voltages required for FEL operation, these accelerators are typically operated in pulse mode at some repetition frequency. The pulse line accelerators are fundamentally single shot machines with pulse times of the order of tens of nanoseconds, while modulators can produce repetitively pulsed beams with pulse times in excess of a microsecond. Electrostatic accelerators can, in principal, produce $\mathrm{CW}$ beams; however, in order to do so it is necessary to recycle the current in the spent beams to replenish the charging current. In practice, $\mathrm{CW}$ operation requires the recovery of better than $99 \%$ of the spent current. This has been done at high efficiency [31] and offers the promise of achieving CW operation [32]. An alternative is to produce the beam near ground potential and have the wiggler at high positive potential and then recover the beam at ground again. Two systems are under test to utilize this approach to produce high average power at $130-250 \mathrm{GHz}$ for heating of fusion research plasmas [33,34] Operation on the third harmonic has yielded 30 micron output with modest voltages [35].

However, operation at wavelengths from the near infra-red IR through the visible requires beam energies in excess of $10 \mathrm{MeV}$. For these applications, the most commonly used accelerator is a conventional copper RF linac. Examples of this technology are the Sband $(2856 \mathrm{MHz})$ Mark III linac at Duke University and its twin system at Vanderbilt University. Using conventional linac technology with injectors that produce $10 \mu \mathrm{s}$ macropulses with a pulse repetition frequency (PRF) of about $60 \mathrm{~Hz}$. To date Vanderbilt holds the average power record for FELs at about $10 \mathrm{~W}$ [36], although several teams are attempting to significantly surpass this record in the near future [37-39]. All produce beams in the infrared with wavelengths ranging 2 to 16 microns.

Electron linacs are typically pulsed copper devices with limited duty factors due to Ohmic heating in the cavities by the microwaves at 0.4 to $3 \mathrm{GHz}$. Higher gradients are generally achievable using higher frequency microwaves. However, the accelerating 
cavities store more energy and have reduced wall interactions (higher order mode production) with the electron beam at lower frequencies. This permits higher average currents and peak charges with less degradation of beam quality than can be achieved with the higher gradients associated with the high frequency cavities. A copper machine at Boeing Aerospace has pushed this technology to its fullest with a $433 \mathrm{MHz}$ accelerator that is capable of $\mathrm{CW}$ operation and has demonstrated a $25 \%$ duty factor. Nearly $130 \mathrm{~mA}$ of high quality macropulse current was produced at over 1 nanocoulomb per bunch [40].

An example of the success that can be achieved with very high energy linacs is the lasing at $278 \mathrm{~nm}$ achieved from the $165 \mathrm{MeV}$ linac at FELI in Japan [41]. Wigglers in this facility have operated at wavelengths from 80 to 0.28 microns. The laser produced $30 \mathrm{~mW}$ in $24 \mu$ s macropulses at $20 \mathrm{~Hz}$ PRF. The lasing achieved here is the shortest oscillator wavelength to date on a linac driven system. A key difficulty is that the FEL gain is dropping at the higher energy at the same time as the reflectivity available from mirrors becomes worse around $225 \mathrm{~nm}$ and shorter where oxide dielectric coatings are no longer useful.

An alternate technology for producing $\mathrm{CW}$ or long macropulses is the superconducting RF linac structure (SRF) typified by the Continuous Electron Beam Accelerator (CEBA) at Thomas Jefferson National Accelerator Facility which produces 4 $\mathrm{GeV}$ electron beams for nuclear physics research using $1497 \mathrm{MHz}$ cavities operated at 2K. Ohmic losses are reduced to negligible levels with the SRF structures (6 W/cavity at typical gradients) while maintaining high acceleration gradients (5 to $18 \mathrm{MV} / \mathrm{m}$ ) [42]. Among many additional factors, the gradient achievable depends on frequency with the higher frequencies producing higher gradients because of the reduced likelihood of a defect occurring over the cavity surface. It is worth noting that the first FEL [43] and the first tapered wiggler oscillator [44] and the first visible lasing on a linac based FEL [45] operated using the Stanford Superconducting Accelerator. Since its original demonstration this linac has been a workhorse serving several generations of FELs since the CW beam 
yields high stability of the power, wavelength, phase, and pulse length. In recent years it has been extremely successful as a user facility producing infrared light for a number of two photon experiments as well as continuing to investigate the physics of the FEL interaction.

Storage rings are a cost effective alternative to producing the energies of up to a few $\mathrm{GeV}$ for operation at wavelengths in the ultraviolet spectrum [46]. Indeed, the shortest wavelength to date produced by an FEL comes from the VEPP3 storage ring at Novosibirsk [47]. One limitation on the storage ring for FEL operation is that interaction heats the electron beam. The time for synchrotron damping in storage rings to cool the beam limits the total rate at which energy can be extracted. This is referred to as the Renieri limit [48]. This limit can be expressed in the form

$$
P_{F E L}=\frac{P_{\text {synch }}}{2 N_{w}}
$$

where $P_{F E L}$ and $P_{\text {synch }}$ denote the power radiated by the FEL and incoherent synchrotron emission, and $N_{w}$ is the number of wiggler periods. While the power radiated by the FEL mechanism is less than the synchrotron emission, it is of interest because it is coherent and emitted over a narrow spectral range.

The challenge in designing storage rings for FELs is to allow for a number of long straight sections for insertion of wigglers. To minimize the effects of debunching from the induced energy spread, modern designs try to make the ring as isochronous as possible. An example of such a system is the recently commissioned storage ring at Duke University [49] that can transport beams of $10 \%$ energy spread without loss. The ring has a circumference of $107.46 \mathrm{~m}$ and provisions for two straight sections, and an energy range of 0.25 to $1.1 \mathrm{GeV}$. The machine is designed to accommodate average currents of up to an Ampere, and has already circulated $8 \mathrm{~mA}$. At the present time two wigglers are installed: the OK4 from Novosibirsk [50] and an undulator originally constructed for a 
microtron-based FEL to have been built at NIST [51]. The OK4 wiggler system lased successfully at $345 \mathrm{~nm}$ beginning in December 1996 producing $150 \mathrm{~mW}$ and small signal gains of nearly $10 \%$. Future plans include operating at much shorter wavelengths and increasing the average power. This system also demonstrated Compton scattering of the FEL light to produce gamma rays at $12.2 \mathrm{MeV}$ (see discussion below). Other FEL storage rings have also achieved notable success: Super-ACO [52], VEPP3 [53], UVSOR [54] , and NIJI-IV [55].

Ultraviolet operation has also been achieved using both high and moderate energy RF linacs [56], and RF linacs are also preferred for X-ray operation because circular machines reach emittance limits due to (1) synchrotron heating of the beam, and (2) the low peak currents due to isochronicity limits. As such, the bulk of FEL user facilities built to date employ RF linacs. The temporal character of the light produced by RF-driven electron sources is noteworthy. All RF machines produce a string of short pulses (micropulses) for a period of time (macropulse) which is then repeated at some pulse repetition rate $(\mathrm{PRF})$, and the output radiation mirrors this pulse structure.

A group at Novosibirsk is pursuing the construction of a high average power system based on a microtron operating with very high average currents and a low RF drive frequency [57]. The 16 accelerator cavities operate at $180 \mathrm{MHz}$ to transport 4 to $50 \mathrm{~mA}$ of current up to $98 \mathrm{MeV}$ and then decelerate the beam again to recover its energy. Rotation of the electron bunch in phase space is performed to minimize emittance growth effects during acceleration and to maximize electron transport down to the dump. Peak currents of up to $100 \mathrm{~A}$ are planned despite starting from a thermionic cathode, and a novel scheme will be used to outcouple the optical energy from the FEL cavity in 10 to 30 ps, 1 to 10 micron pulses.

\section{B Injectors}


No part of the FEL design has more influence on the eventual performance of a linac driven system than the injector. Significant strides in that technology over the past decade have influenced linac design for a wide range of applications not restricted to FELs. Most notable has been the development of the RF photocathode injector. Pioneered by Sheffield and Carlsten at Los Alamos National Laboratory [58], this technology permits the production of electron bunches with nanocoulombs of charge at emittances of several mm-mrads. Such performance has yielded ultraviolet lasing using electron beam energies of only 45.2 MeV [56]. Emittance growth in these injectors can be reduced by the proper design of the electrostatic and/or rf focusing system to compensate for the major sources of emittance growth (primarily space-charge effects) [58]. The high brightnesses at high bunch charge are also significantly dependent on the high cavity gradients achievable in pulsed structures. Typically a minimum of 20 to $40 \mathrm{MV} / \mathrm{m}$ is desired on the photocathode surface although operating gradients up to $125 \mathrm{MV} / \mathrm{m}$ at the cathode have been reported [59].

This photo-injector technology is a key enabling technology for the development of X-ray FELs based on Self Amplified Spontaneous Emission (SASE). Such FELs require very small emittances to work at the short wavelengths desired and high peak currents and bunch charges in order to get sufficient gain to keep wiggler lengths within reason. Typically these injectors produce more than 1 nanocoulomb pulses which could lead to severe emittance degradation by space charge forces and phase space mixing were it not for the high acceleration gradients which get the beam to relativistic energies quickly (in addition to a solenoidal focusing field). Groups at BNL, UCLA, and SLAC are all collaborating on such a photoinjector [60]. The effort is aimed at producing 1 nanocoulomb in a 13 ps flat-top pulse with a projected normalized emittance of $<1.25$ $\mathrm{mm}-\mathrm{mrad}$ at $30 \mathrm{MeV}$ using gradients up to $150 \mathrm{MV} / \mathrm{m}$. BNL has already demonstrated 2.5 $\pm 0.5 \mathrm{~mm}$ mrad at 1 nanocoulomb in a $10 \mathrm{ps}$ pulse [59]. 
A technical challenge in the design of photoinjectors is the need for ultrahigh vacuum to avoid poisoning of the cathode material. It is especially difficult to engineer excellent pumping speeds in very high gradient cavities so care in surface preparation is required. Vacuums of $10^{-9}$ to $10^{-10}$ are required for most cathode materials with water vapor being a key poisoning element. Typically partial pressures of $10^{-11}$ of $\mathrm{H}_{2} \mathrm{O}$ are desired to maintain high quantum efficiency (up to 15\%). Even when the cathode is not poisoned by an imperfect vacuum, then back bombardment by ions onto the cathode surface can result in lifetime limitations. Lifetime is governed then by total integrated charge delivered rather than time.

The laser source can be doubled, tripled, or quadrupled YAG or YLF depending on the cathode material. A number of different materials have found favor at different institutions: $\mathrm{Cs}_{2} \mathrm{Te}, 13 \%$ QE @ 263 nm with lifetimes of 100 s of hours, $\mathrm{LaB}_{6}, 0.1 \%$ @ $355 \mathrm{~nm}$ with lifetimes of 24 hours, $\mathrm{K}_{2} \mathrm{CsSb}, 8 \%$ QE @ $527 \mathrm{~nm}$ with lifetimes of 4 hours, Cs33 Sb, 4\% QE @ 527 nm with lifetimes of 4 hours, and GaAs (Cs), 5\% QE @ 527 nm with lifetimes $>40$ hours (see [61] and [62] for a review of many cathode materials). The lifetime data quoted here should be taken with some degree of skepticism since no attempt has been made to unfold the effect of delivered charge and therefore back bombardment of the cathode life. Some cathode materials can be rejuvenated many times with oxygen cleaning and/or re-cesiation. Often injector designs incorporate a means to prepare or transfer new cathodes to the cavity or alternatively a cassette with multiple cathodes.

No ideal photocathode exists which combines long life, insensitivity to poisoning, high quantum efficiency, and long wavelength operation. A few materials with relatively low quantum efficiency have essentially infinite lifetime (e.g., $\mathrm{LaB}_{6}$ run very hot but below the threshold for thermionic emission). The cathodes with the best life are those which require UV light which requires more fundamental power because of the lower conversion efficiency in quadrupling although conversion efficiencies of $30 \%$ have been achieved. Achieving the desired stability in phase and amplitude and reliability in the drive laser is 
also not trivial. It is particularly challenging if high duty factor operation is desired as on SRF linacs; the resulting lasers tend to be at the edge of commercial availability and affordability.

To achieve CW operation from injectors requires either a substantial RF system and aggressive cavity cooling design [38], a DC photogun development [63], or use of a thermionic cathode with a concomitant increase in the emittance at a given charge [57]. To date, no SRF photogun has been developed beyond some low current demonstrations [64] although such a development would have significant potential applications. A group at Rossendorf is pursuing such a development [65]. They believe it is possible to achieve nearly $20 \mathrm{MV} / \mathrm{m}$ on the cathode and $10 \mathrm{MV} / \mathrm{m}$ average in the cavity in a TESLA-style 3 1/2 cell $1300 \mathrm{MHz}$ cavity and are constructing a 1-1/2 cell prototype. There are no fundamental physics issues identified but the engineering challenges are significant.

Thermionic RF guns are also commonly used for compact FELs. Such systems can share the RF power from the main linac structure with appropriate phase and amplitude control on the RF. Because the electrons can be emitted over a broad range of RF phases the electrons have a wide energy spread. With such systems the beam is generally sent through an alpha magnet with a slit defining the energy acceptance for the rest of the machine. Such systems are in operation in Duke [66], Vanderbilt [67], IHEP [68], and elsewhere. To help RF phase and amplitude control, feed-forward circuits can be used to anticipate the beam loading effects.

\section{Wigglers}

The wiggler or undulator represents a mature technology with several excellent designs available from a number of commercial companies. It should be explained that the difference between wigglers and undulators is determined by the value of $K(\sim 0.934$ $B_{w} \lambda_{w}$ where $B_{w}$ is the on-axis wiggler field amplitude in Tesla and $\lambda_{w}$ is the period in $\mathrm{cm}$ ), as referred to by $a_{w}$ previously in this paper and in much of the literature in the field. In the 
FEL community these terms are used interchangeably, but in the synchrotron light source community the term undulator is used more commonly when $K \leq 1$. Wigglers have been constructed with both helical and planar symmetry, and with electromagnets, permanent magnets, or hybrid combinations of the two. Ferrite elements are also used to concentrate the field. The commercial success of these devices has been due not so much by the market drive from the FEL community but rather that of the second and third generation synchrotron light sources which can have many insertion devices and where the required quality of the magnetic field is very high.

The technology of choice is wiggler period dependent. For microwave/millimeter wave applications electromagnetic wigglers prevail, and both helical and planar wiggler configurations have been used with wiggler periods ranging from 3-10 cm. At wavelengths in the near infrared and shorter, planar wigglers are most commonly used. Examples of an electromagnet and an electromagnet with ferrite elements to confine the field include the PALADIN wiggler built at Lawrence Livermore National Laboratory [69], the FIREFLY wiggler at Stanford's SCA [70], and the OK4 wiggler from Novosibirsk now installed at Duke [71]. For wiggler periods of $6 \mathrm{~cm}$ down to $2 \mathrm{~cm}$ or less permanent magnet/hybrid wiggler technology takes over utilizing $\mathrm{SmCo5}$ or $\mathrm{NdFeB}$ permanent magnets with flux channeled by vanadium permendur or similar materials to produce $K^{\sim} 1$ for approximately $1 \mathrm{~cm}$ gaps. These are extensions of a technology originally developed by Halbach [72], and are sufficient for significant gain in the infrared and visible spectra. One typical example is the Jefferson Lab IR Demo wiggler manufactured by STI Optronics which has a $K=1$ at a $12 \mathrm{~mm}$ gap with a $2.7 \mathrm{~cm}$ wavelength and 40.5 effective periods. This wiggler was based on a design for an insertion device at the Advanced Photon Source at Argonne National Laboratory and exhibits exceptionally good field quality. It is important to note that it is now recognized that the key field quality requirements are the phase error and the trajectory error. The previously used RMS field error, while marginally useful, is not nearly as traceable to FEL performance [73]. 
To obtain shorter wiggler periods at reasonable gaps becomes difficult since the field falls exponentially as the ratio of the gap to the wavelength. Pulsed electromagnetic [74], super-ferritic (superconductor with iron poles) [75,76], and superconducting [77] systems have been built, as well as very short wavelength hybrid or permanent magnet devices with small gaps. No system has shown clear superiority for all applications at this time. Tunability is achieved by varying either the electron beam energy or the field strength. If the wiggler is adjustable then it is much easier to tune the wavelength since electron transport systems are chromatic and require retuning if the beam energy is adjusted outside a narrow range. Tuning electromagnetic wigglers is simply a matter of adjusting power supplies while tuning hybrid wigglers requires one to adjust the gap. Tolerances are tight and the since trimming of the field quality can only be optimized at one gap some penalty in performance may result when utilizing a wide tuning range.

One consideration in the choice of wigglers which comes into play for long wigglers is the necessity of maintaining the electron beam focus. At low electron beam energies this can be done via an imbedded solenoidal guide field but this becomes impractical above a few $\mathrm{MeV}$ in electron beam energy. Helical wigglers provide focusing in both planes (and higher gain by root (2) ) but are difficult to manufacture and trim at high fields. They also provide little access to the beam for diagnostics or vacuum pumping. The more common planar wigglers naturally provide focusing in the direction of the magnetic field which can maintain one beam axis at a stable value through the full length of the wiggler if properly matched initially. If nothing further is done there is neutral focusing action in the other plane. This may be acceptable for short wigglers but additional focusing is required if the wiggler is long. Such focusing can be provided by providing distributed quadrupole fields by curved poles [5] or permanent magnets [78-80], or occasionally adding quadrupoles interspersed between wiggler sections. The tolerances can be very tight in the case of very long SASE systems. There is substantial engineering design and analysis work underway to solve this issue $[81,82]$. 


\section{The Optical Cavity}

The optical cavity for an FEL is often more difficult to engineer than for conventional lasers. The FEL requires good overlap between the electrons and the optical mode in order to achieve high optical field amplitudes. Since the electron beam dimensions are small, this implies that the mode must also remain small with a relatively short Rayleigh range but modest mode size variations within the wiggler. A broad performance optimum occurs with the Rayleigh range around half the wiggler length. Angular alignment tolerances can be very small. In addition, the cavity length must match a subharmonic of the linac operating frequency (or the interpulse spacing in a storage ring) to a very high accuracy. It is not unusual to require a $10 \mathrm{~m}$ optical cavity length to be correct to within a few microns. The range over which the optical cavity can be varied and still result in lasing is called the detuning length. It becomes particularly small when pulse lengths are short and system gains drop as happens in systems designed for short wavelength operation. This tends to not be such a problem in storage ring devices because of the long micropulse lengths although the typically lower gains still result in tight angular tolerances and high cavity Qs. Since the optical output mimics the electron beam's temporal microstructure, a subset of possible optical cavity modes is excited and therefore contributes to the output [83]. The optical cavity must operate in a vacuum and usually must be remotely controlled because of the radiation environment. The high Qs and tight optical modes typically found yield high peak and average powers on the optics which can lead to damage as can the more gradual background radiation from the accelerator. Higher energy machines produce significant fluxes of hard UV at the FEL harmonics which can lead to mirror damage [84-86]. Moreover FEL designers would generally prefer to have mirrors of high reflectivity over a broad wavelength range to take advantage of the FEL's tunability. Such mirrors are somewhat obtainable in the IR region but coating performances become worse as one moves toward the UV. Outcoupling the power 
requires a transmissive optic (which may be difficult over some wavelength bands), hole outcoupling [87], which results in only part of the power going into a useful mode although see the novel RAFEL design discussed below), an unstable resonator design with a scraper (extra mirror bounces), a grating (perhaps difficult to manufacture or have survive at high fluence), or use a two stage system with the high power produced in an amplifier stage at the end (promising but the difficulties have not yet been fully explored).

Given all these design issues it is not surprising to find a number of different approaches having been taken. Short wavelength or high power operation places especially high demands on the cavity and novel approaches have been chosen to deal with such issues [88-90].

\section{Important Future Directions}

At the present time, $10 \mathrm{FEL}$ user facilities are in operation for a wide range of applications in materials and bio-medical research as well as a large number of dedicated FELs (for a listing see [91] for short wavelength FELs and [92] for long wavelength systems). While it is always hazardous to try to predict the future course of scientific research, we feel confident that two general areas of research will comprise important elements of future study. These two areas include advances in the technology to achieve (1) higher average powers, and (2) shorter wavelengths. In the first case, the initial target will be to achieve average powers of $1-10 \mathrm{~kW}$ in the infrared. This goal is under aggressive pursuit at the Thomas Jefferson Accelerator Laboratory where a superconducting linac is being built to power an FEL oscillator. In the second case, designs are being finalized for short wavelength SASE FELs at SLAC and DES to use RF linacs to generate beams with energies in excess of $1 \mathrm{GeV}$ to reach wavelengths $60 \AA$ or less with brightnesses greater than can be achieved with synchrotron light sources. Indeed, these SASE FELs are, at the present time, the prime candidates for the fourth generation light source. 


\section{A. SASE for Short Wavelengths}

One of the more active areas in the past few years has been in developing an approach to producing a soft X-ray FELs with wavelengths near the $40 \AA$ "water window" so a hologram of a (formerly) living protein could be obtained, or near $1.5 \AA$ so a holographic image at the atomic scale could be obtained. Difficulties that must be overcome include (1) the requirement for extremely low emittance beams, (2) the lack of a suitable source for an amplifier, (3) the lack of good mirrors for oscillators, and (4) the relatively low growth rates result in extremely long wigglers with concomitant tight alignment tolerances, required for a SASE configuration. The typical design under consideration for these systems is the SASE FEL to avoid the requirements for an external source or good X-ray mirrors. Here, shot noise [93] in the initial electron bunch provides the initial photons, typically having an equivalent power of 10 to 1000 watts due to the large electron charges involved [94]. However, the short wavelength required demand relatively high electron energies, and most designs involve beam energies of $1 \mathrm{GeV}$ or more.

A design underway at SLAC will serve as an illustration which is based on using $1 / 3$ of the SLAC linac at $15 \mathrm{GeV}$ with an RF photocathode source for a high brightness beam. A schematic illustration of this design is shown in Fig. 9. The injector produces 1 nanocoulomb in a 3 ps bunch which is accelerated and compressed using magnetic bunching in three stages to 3400 A peak in a 100 fs pulse at $1.5 \mathrm{~mm}-\mathrm{mrad}$ emittance. Such a pulse would have a gain length of approximately $11 \mathrm{~m}$ in a $K=3.7,3 \mathrm{~cm}$ period wiggler, and a wiggler length of $94 \mathrm{~m}$ is needed to reach saturation. The short electron pulse length is achieved by a magnetic bunching chicane and a correlated energy spread on the beam. At the present time, there are unanswered questions about the growth of emittance in such chicanes due to coherent synchrotron emission effects which could lead to performance problems [95]. Efforts are underway to answer this outstanding question from both theoretical and experimental standpoints. A peak output power of $10 \mathrm{GW}$ is indicated at a 
wavelength of $1.5 \AA$. Key challenges include maintaining the required emittance $(1.5 \mathrm{~mm}$ mrad), achieving low dipole field errors, achieving very tight alignment (absolute beam trajectory deviation $<5$ to 10 microns) [96]. Other work is aimed at developing alternate approaches that reduce the formidable hardware requirements [97].

A similar concept, but based on advanced superconducting structures for TESLA is under construction at DESY [98]. By taking advantage of the reduced perturbations from longitudinal and transverse wakefields the plan is to reach a wavelength of $65 \AA$ using a $1 \mathrm{GeV} / 2500$ A electron beam and a wiggler with an on-axis field strength of $5 \mathrm{kG}$ and a period of $2.73 \mathrm{~cm}$. Saturation is anticipated over a wiggler length of about $27 \mathrm{~m}$ with a peak output power of $2-3 \mathrm{GW}$. The TESLA Test Facility has a high duty factor for high average brilliance (see Fig. 10). The figure shows the output of the Tesla Test Facility (TTF-FEL) at $1 \mathrm{GeV}$ under conditions of straight amplification and with the addition of a monochromator at the midpoint of the wiggler to increase the brilliance (TTF-FEL M). Later the full upgrade to $50 \mathrm{GeV}$ will result in the output shown as TESLA-FEL. An alternative to this project using S-band normal conducting accelerators is shown as SBLC. The SLAC Laser Coherent Light Source (LCLS) is shown for comparison.

Such systems depend on the success of SASE to achieve their goals but at the present time only limited demonstrations of SASE have been achieved. Work in the long wavelength regime at LLNL on the ELF system produced high extraction efficiency and very high gains [99] but this was in a waveguide geometry so that the connection to present plans is not as strong as desired. More recent efforts have demonstrated SASE at wavelengths in the vicinity of 600 microns [100] at the Massachusetts Institute of Technology was in substantial agreement with theoretical predictions. Recently, SASE has been clearly demonstrated at 16 microns [101]. In addition, the equipment is in hand for a SASE experiment at Duke University using the PALADIN wiggler [69] and the injector 
linac for the storage ring, and a conceptual design for such an experiment has been developed at a wavelengths of about 1.4 microns [102].

Theoretical efforts [93] predict the production of short, intense spikes within the micropulse separated by the effective cooperation length. This is similar to effects seen in oscillators at high gain $[103,104]$. Whether these intense spikes will prove useful or a hindrance is an open issue.

\section{B. Superconducting RF Systems}

An approach to producing high average powers and efficiency improvements involves the recovery of electron beam energy by decelerating the electron beam in the same cavities used for the initial acceleration. Although FEL lasing has previously been accomplished while decelerating the beam in a second accelerator [105] and same cell energy recovery has been done in the absence of FEL operation [106], no complete demonstration of these techniques has yet been accomplished. A group at Jefferson Laboratory is incorporating such an approach into a high average power FEL for industrial processing [37]. A schematic illustration of this FEL is shown in Fig. 11. The system will operate initially at $1 \mathrm{~kW}$ in the $3-6.5$ micron range. Later upgrades are

planned to take the system to 1 micron and later to $2000 \AA$. The average beam current of $5 \mathrm{~mA}$ operates $\mathrm{CW}$ because of the superconducting accelerator cavities. In its initial 3 micron configuration the $10 \mathrm{MeV}$ injected beam is accelerated to $42 \mathrm{MeV}$, passed through the wiggler to achieve lasing, and then decelerated to the injection energy. The difficulty, of course, is the energy spread induced by the FEL. High transport acceptance is essential since on a CW basis little more than a few microamperes can be permitted to intercept the beamline wall before melt through would occur. Just how large the extraction efficiency can be before the beam becomes uncontrollable is the subject of ongoing study. There are also open issues regarding the system stability since fluctuations in the accelerated and 
decelerated beam can cause beam energy variations affecting the lasing. The system construction is completed and first lasing is planned in FY98.

\section{RAFEL}

In an effort to extend the efficiency of oscillators and simultaneously deal with the ever present issue of mirror loading, a group at LANL has proposed using a high gain amplifier with a hole coupling resonator which takes advantage of the fact that the oscillator mode tightens as the system comes into saturation. Thus, at low powers most the radiation is fed back to be amplified whereas at high powers most of the optical power flows through the hole and is outcoupled and only $1 \%$ of the power is fed back at saturation. This reduces heating and consequent damage of the mirrors. The system requires significant gains and so puts significant demands on the electron beam quality at shorter wavelengths. A demonstration experiment is underway operating at 16 microns. It has already shown small signal gains of 60 using the nominally $17 \mathrm{MeV} 300$ A pulses 20 ps long in 30 microsecond macropulses at $60 \mathrm{~Hz}$. The system is expected to ultimately provide $1 \mathrm{~kW}$ of average power [15].

\section{Miscellaneous Approaches.}

Other groups are extending the FEL performance in other ways which will provide users desirous of special photon characteristics more flexibility. FEL operation has already demonstrated very narrow line operation by utilizing coupled cavities to tighten the spectrum [107]. Methods have also been found which permit the FEL to lase at multiple frequencies simultaneously, either near by [108] or on harmonics [109] or with synchronous use of synchrotron emission [110]. To deal with the high optical cavity power a group has proposed using an initial low power oscillator to bunch the beam which then provides high gain and extraction in a second wiggler [57]. Such a concept differs from earlier work on a MOPA (Master Oscillator Power Amplifier) [111] in that the 
electron transport to the second wiggler must maintain the electrons' phase coherence to the optical wavelength accuracy and so be of exceptionally high quality.

Although not strictly an FEL question, there have been a number of papers and experiments in the last two years utilizing FELs or conventional laser sources for Compton scattering to promote photon energies up to MeV levels. Such sources could have application in medicine [112], nuclear physics research [113,114], or the study of transmutation of elements [115]. An FEL is particularly advantageous as the initial photon source for this because the light pulses are of high intensity being within the optical cavity, and are automatically matched in temporal and physical alignment with the electron beam. The FEL is operated with more than one optical bunch in the laser cavity so that the returning optical pulse encounters a electron bunch and scatters up to an energy given by $h \omega=4 \gamma^{2} h \omega_{0} /\left(1+\gamma^{2} \theta^{2}\right)$. Note that additional corrections are necessary if the photon energy is comparable to the rest mass of the electron [116]. At high photon energies the electrons are lost after the scattering event due to the recoil momentum bringing the electron outside the storage ring acceptance. One key factor in the experimental design is making sure the interaction of the electrons and photons occurs at a point where the electron angular dispersion is smallest, i.e. field free. To date a number of groups have been successful in generating such photons using storage rings and linacs at UVSOR to produce $9.5 \mathrm{MeV}$ photons [117], Duke to produce $32 \mathrm{MeV}$ [118], CLIO at 7 to $14 \mathrm{keV}$ [119], etc.

\section{Applications}

As the FEL has matured there has been a substantial growth in the number of efforts to utilize the radiation produced rather than just study it. It is impossible to cover all areas of research underway or contemplated using FELs but we will try to cover many broad categories to illustrate the advantage of an FEL source. Indeed, it must be recognized that to be successful as a research source, the FEL must offer capabilities not 
found elsewhere for this is necessary to overcome the clear disadvantages of FEL sources: large, expensive, requires a "crew" to run as opposed to a tabletop turnkey system. Several studies have examined the applicability of FELs to perform basic scientific research [120] and have found wavelength ranges in which the FEL has no effective competition (10 microns to several $\mathrm{mm}$ excepting several fixed points where existing lasers can operate). In other regions it may still be the source of choice by virtue of other characteristics: short pulse length, micropulse energy/peak power, ability to synchronize for pump probe efforts, bandwidth, beam quality, and, most fundamentally, tunability.

\begin{tabular}{|c|c|c|c|c|c|c|c|c|}
\hline Country & $\begin{array}{l}\text { Instituti } \\
\text { on }\end{array}$ & Device & $\lambda(\mu \mathrm{m})$ & $\tau_{p}(\mathrm{ps})$ & $\begin{array}{l}E_{b} / I_{b} \\
(\mathrm{MeV} / \mathrm{A})\end{array}$ & $\begin{array}{l}\text { Ppeak } \\
\text { (MW) }\end{array}$ & $\begin{array}{l}P_{\text {avg }} \\
\text { (W) }\end{array}$ & $\begin{array}{l}\text { Acceler } \\
\text { ator }\end{array}$ \\
\hline \multirow[t]{8}{*}{$\overline{\mathrm{USA}}$} & \multirow[t]{2}{*}{ 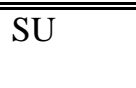 } & "FIREFLY & "19-65 & 10 & $20 / 14$ & $\bar{~} .3$ & 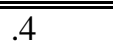 & "SCRF \\
\hline & & SCA/FEL & $3-10$ & 0.7 & $37 / 10$ & 10 & 1.2 & SCRF \\
\hline & VU & FELI & $2-10$ & 2 & $43 / 50$ & 10 & 10 & RTRF \\
\hline & \multirow[t]{2}{*}{ DU } & Mark III & $3-10$ & 3 & $44 / 20$ & 2 & 3 & RTRF \\
\hline & & OK-4 & 0.3 & 10 & $1000 / 350$ & 1000 & 0.1 & SR \\
\hline & $\mathrm{JL}$ & IR Demo & $3-6.6$ & $1-2$ & $42 / 50$ & (10) & (1000) & SCRF \\
\hline & UCSB & & $150-2000$ & $6 \infty 10^{6}$ & $6 / 2$ & 0.004 & 0.08 & VDG \\
\hline & PU & CIRFEL & $10-43$ & 4 & $14 / 150$ & 4 & 0.05 & RTRF \\
\hline \multirow[t]{5}{*}{ Japan } & \multirow[t]{5}{*}{ FELI } & FELI 1 & $5-22$ & 1.7 & $33 / 42$ & 5 & 2 & RTRF \\
\hline & & FELI 2 & $1-6$ & 1.7 & $75 / 50$ & 5 & 0.5 & RTRF \\
\hline & & FELI 3 & $0.23-1.2$ & 1.7 & $165 / 60$ & 5 & 0.5 & RTRF \\
\hline & & FELI 4 & $20-80$ & 1.7 & $30 / 40$ & 5 & 1 & RTRF \\
\hline & & FELI 5 & $40-100$ & $3-5$ & $20 / 40$ & (2) & (1) & RTRF \\
\hline \multirow[t]{2}{*}{ France } & \multirow[t]{2}{*}{ LURE } & SuperACO & 0.3 & 20 & $800 / 10$ & 12 & 0.8 & SR \\
\hline & & CLIO & $1.8-17.5$ & $1.5-6$ & $70 / 80$ & 10 & 9 & RTRF \\
\hline \multirow[t]{2}{*}{ NL } & \multirow[t]{2}{*}{ FOM } & FELIX-2 & $5-35$ & $0.5-10$ & $45 / 70$ & 2 & 1 & RTRF \\
\hline & & FELIX-1 & $25-110$ & $1-10$ & $25 / 70$ & 2 & 0.5 & RTRF \\
\hline China & Beijing & BFEL & $10-16$ & 4 & $30 / 14$ & $\overline{20}$ & 2 & RTRF \\
\hline
\end{tabular}

Table 1 Summary of currently available FEL user facilities. See [91,92] for references.

There are at least 10 major multi-user FEL facilities around the world. In the last year they have collectively provided over 14,000 hours of beam time to researchers. They are applying light for research in material sciences, chemical technology, biophysical science, medical applications, surface studies, and solid state research. Despite this active agenda, the number of FEL users does not approach that of synchrotrons and other such light sources. This is primarily due to the fact that the FEL supports more or less a single 
user at one time. While it is feasible to split the FEL's output beam among several groups, all must agree on the wavelength, pulse length, pulse structure, etc., which is an unlikely event in most cases. Therefore, except for parasitic alignment activities, the FEL will continue to support single user activities for the most part. An exception to this is the FEL facility at Stanford which has installed a system for beam separation to permit sending the independently adjustable beam to alternate wigglers on a macropulse basis [121].

The FEL applications span a wide wavelength range and the sorts of applications that occur in each range are treated below.

\section{A. Millimeter Wave/Far Infrared Spectra}

At least three groups are attempting to make high average power FELs in the $\mathrm{mm}$ range for plasma heating. In this frequency range gyrotrons have been the principle radiation source. The FEL offers possibilities of better performance at the higher frequency ranges of interest because the source produces a quasi-optical mode. This is easier to produce and transport without overtaxing windows and other RF components. The FEL can also excel as a research tool in this range because it produces ps pulses of high intensity radiation. Typical studies include measurements of principle excitations in condensed matter systems where it is possible to access the principal excitations such as plasmons, phonons, magnons and inter-sub-band transitions. Direct linear probing of defect modes and buried interfaces with bond specificity is possible [122]. Measuring energy distributions and line shapes probes mode coupling and energy dissipation into the electron or phonon continuum of the substrate. Low frequency modes in large biomolecules such as nucleic acids and proteins can be excited in FIR for similar studies of energy flow.

An interesting byproduct of research in this area is the development of a far infrared streak camera capable of operation out to 100 microns with picosecond resolution [123]. 


\section{B. Infrared Spectra}

Most existing FELs operate in this wavelength range. For many uses at wavelengths less than around 10 microns existing tabletop lasers can satisfy most researchers needs due to the development of OPOs for nanosecond and OPAs for picosecond pulses. These use b-barium borate $(\mathrm{BBO})$, potassium trihydrogen phosphate (KTP), lithium niobate (LiNbO3), and AgGaSe2. Such systems span $440 \mathrm{~nm}$ to 2 microns with $10 \mathrm{~ns}$ pulses producing 20 to $100 \mathrm{~mJ}$ of light and from 1 to $10 \mathrm{~mJ}$ at 2 to 3.5 microns.

Exceptions which drive users to FELs occur when they need high peak powers, have a particular need to exploit the wide tunability of the FEL, or the possibility of chirped pulses. OPOs also tend to have bandwidths which are wide compared to their Fourier width. Despite the competition this has been a fruitful range for researchers desiring to utilize the FEL. Many of the researchers synchronized the FEL output with another radiation source for measurements in this wavelength range: Stanford used Ti:sapphire; the Mark III and Duke storage ring used synchrotron emission; at CLIO they used frequency doubled mode locked YAG.

A particularly powerful technique has been sum frequency generation. The versatility of this approach is demonstrated by the variety of applications demonstrated. Early examples at CLIO include sum-frequency measurements on the surface of Pt in methanol using 5 micron FEL pulses and a synchronized laser. In other tests the FEL was used for pump probe observation of coherent transient grating effects of narrow gap semiconductors and third order nonlinearity coefficients and electron relaxation times in GaAs/AlGaAs quantum wells [124], More recent activities include using SFG to identify bonding and density monitoring when putting polyurethane on float glass, interaction of fullerene deposited on gold to show the surface interaction modifies the fullerene geometry and vibrational dynamics of $\mathrm{CO}$ at an electrochemical interface [125]. 
Studies in kinetics in this wavelength range include vibrational energy transfers in molecules. This opens up a new class of experiments to study mode selective chemistry that requires high power short pulses which excite molecular vibrations. For example see [126] for isotope selective multiphoton dissociation of formic acid and nitromethane

A group at Stanford studied second order nonlinear susceptibility of the conduction band and valence band quantum well (QW) structures extracted from the interference between second harmonic fields of QWs and GaAs substrate as determined by the azimuthal dependence of the second harmonic power. This is the first demonstration of difference frequency generation of mid IR in any QW [127].

Groups at Stanford also studied vibrational dynamics in glass forming liquids. These are the first vibrational photon echo experiments and first comprehensive temperature dependent pump probe measurements on any condensed matter system [128].

Among the extensive studies carried out at the FELI facility in Japan are resonant excitations of molecular vibrations [129], band discontinuities of semiconductor heterojunctions [130], and isotope separation [131]. The user facilities at this lab have become among the most productive in the world, providing over 2000 hours of beam time in the last year.

Studies in medical applications at Vanderbilt are leading in 1998 to the first use of an FEL in human neurosurgery by taking advantage of particular absorption bands to produce an exceptionally fine cut in bone and tissue [132]. FELI has also ablated and hardened dental materials [133] and studied photodynamic therapy [134]. The tunability, power, and pulse variability of the FEL has made it an efficient biophysical research tool [135].

Commercial applications have been proposed which use a high average power FEL to heat the surface of polymers for enhancements to the surface morphology. This uses infrared at 5.8 to 6.2 micron wavelengths where high absorption results from carbonylrelated molecular absorption bands. Representing a major market, they would plan to treat 
many billion lbs/yr. The key is to achieve very high average power $(100 \mathrm{~kW})$ at reasonable costs per photon $(<\$ 0.01 / \mathrm{kJ})$. By enhancing surface roughness in polyester and nylon fibers, the fabrics can be made softer, hydrophilic, and the material more readily accepts dyes [136].

\section{Visual/Ultra-Violet Spectra}

Although existing lasers in this wavelength region satisfy most users there were a few applications cited which apply the unique advantages of the FEL. At SuperACO, operating at an average power of $0.1 \mathrm{~W}$ in the $350-430 \mathrm{~nm}$ range time resolved polarized fluorescence decays of the reduced nicotinamide adenine dinucleotide coenzyme NADH in aqueous solution using single photon counting were performed. The advantage of using an FEL on a storage ring is the natural synchronization with synchrotron emission in bending magnets. This was used to study relaxational dynamics of the excited state. In other experiments, the measured the surface photovoltage effect on $\mathrm{Si}$ (111) and the resulting modification of electronic band bending using time resolved photo emission [137].

Current efforts include photo-ionization of excited helium, FEL excitation of photocarrier and production of photofragments in IR, and transient absorption in excited tumor cells [110].

Some proposals for commercial and other applications have been floated and are under study. For example, power beaming to satellites $[138,139]$. The idea is to provide enhanced light intensity to increase the efficiency of solar panels. Proponents claim an improvement of $1000 \%$ more electricity per panel than from the sun which saves $\$ 0.5 \mathrm{~B}$ per satellite slot in a geosynchronous orbit.

Other commercial possibilities are under study. For example, processes have been identified for existing UV lamps and excimer lasers. The difficulty with conventional sources is the cost per $\mathrm{kJ}$ and the available power per unit. This represents an opportunity for the FEL if the desired wavelength ( $200 \mathrm{~nm}$ ) can be produced at high average power 
$(100 \mathrm{~kW})$ and reasonable cost. The high power requirement comes from the need to treat $1000 \mathrm{~m}^{2} / \mathrm{min}$ and deliver $0.5-3 \mathrm{~J} / \mathrm{cm}^{2}$. A patent exists for anti-microbial nylon with wide application if suitable source characteristics can be obtained [140].

\section{Deep Ultra-Violet and X-Ray Spectra}

In the deep UV range the FEL competes with synchrotron sources. Typically, synchrotrons provide average, not peak power, and the pulses are $10 \mathrm{~s}$ of ps in duration at $1 \mathrm{~ns}$ intervals as opposed to the few picoseconds of FELs. FEL oscillators also provide a narrow linewidth so when peak power at a particular wavelength is desired the FEL will hold the edge and the synchrotron will have the advantage for average powers of wide bandwidth. While a number of possible applications have been identified for such sources no FEL yet has the necessary radiation characteristics.

In the X-ray region competition is from laser pumped X-ray sources. A number of workers in the field believe the FEL will be the 4th Generation light source to use in probing at the atomic scale. Applications would involve flash X-ray microscopy, measurements of transient lattice distortions and melting, microbe analysis, imaging, tomography, near resonant scattering, angiography, lithography.

Compton scattering in an FEL provides $\mathrm{MeV}$ level potentially polarized photons which are useful probes of nuclear matter [141] or therapy [142]. This is likely to prove a fruitful area for future research.

\section{Summary}

In this brief survey of free-electron lasers, we have tried to convey of the basic physics, engineering, and applications of FELs, as well as the principal future directions of the field. Of course, this last subject is highly subjective and represents the best judgment of the authors at the present time. As a result, the reader should be aware that future developments may take alternate directions which are not presently apparent. Be that as it may, however, the reader should be aware that applications of this technology abound 
and that, unlike other vacuum electronic devices, the FEL is capable of operation over virtually the entire electromagnetic spectrum.

\section{Acknowledgements}

This work was supported in part by the Office of Naval Research ,and in part under Navy/DOE Interagency Agreement No. 1846-J053A1 and DOE Contract No. DEAC05-84ER40150.

\section{References}

1. This is updated from a plot which appeared in R.K. Parker and R.H. Abrams, Jr. "Radio-frequency vacuum electronics: a resurgent technology for tomorrow", in Millimeter Wave Technology IV and Radio Frequency Power Sources, J.C. Wiltse and J.T. Coleman, eds. Proc. SPIE, vol. 791, pp. 2-12, 1987.

2. H. Motz, "Applications of radiation from fast electron beams", J. Appl. Phys., vol. 22, pp. 527-535, 1951.

3. H. Motz, W. Thon, and R.N. Whitehurst, "Experiments on radiation by fast electron beams", J. Appl. Phys., vol. 24, pp. 826-833, 1953.

4. H. Motz and M. Nakamura, "Radiation of an electron in an infinitely long waveguide", Ann. Phys., vol. 7, pp. 84-131, 1959.

5. R.M. Phillips, "The ubitron, a high power traveling-wave tube based on a periodic beam interaction in an unloaded waveguide", IRE Trans. Electron Dev.,vol. ED-7, pp. 231$241,1960$.

6. R.M. Phillips, "History of the ubitron", Nucl. Instrum, Meth., vol. A272, 1-9, 1988.

7. George R. Neil and the Jefferson Lab FEL Team, "Industrial Applications of the Jefferson Lab High-Power Free-Electron Laser", to be published in Beam Interactions with Materials and Atoms, Nucl. Instru. Meth. B, 1998.

8. H.P. Freund and T.J. Antonsen, Jr., Principles of Free-electron Lasers (Chapman \& Hall, London, 1996), 2nd edition. 
9. M. Cornacchia et al., "Performance and design concepts of a free-electron laser operating in the x-ray region", in Free-Electron Laser Challenges, eds. P.G. O'Shea and H. Bennet, Proc. SPIE, vol. 2988, pp. 2-14, 1997.

10. J. Rossbach et al., "A VUV free-electron laser at the TESLA test facility at DESY", Nucl. Instrum. Meth., vol. A375, pp. 269-273, 1996.

11. N.M. Kroll, "Relativistic Synchrotron radiation in a medium and its implications for stimulated electromagnetic shock radiation", in Physics of Quantum Electronics: Free-Electron Generators of Coherent Radiation, eds. S.F. Jacobs, H.S. Pilloff, M. Sargent, M.O. Scully, and R. Spitzer (Addison-Wesley, Reading, Massachusetts, 1980), vol. 7, pp. 355-375.

12. H.P. Freund, P. Sprangle, D. Dillenburg, E.H. da Jornada, B. Liberman, and R.S. Schneider, "Coherent and incoherent radiation from free-electron lasers with an axial guide field", Phys. Rev. A, vol. 24, pp. 1965-1979, 1981.

13. K.J. Kim, "Characteristics of synchrotron radiation", in The Physics of Particle Accelerators, eds. M. Month and M. Dienes (American Institute of Physics, New York, 1989), AIP Conf. Proceedings No. 184, pp. 565-632.

14. A. Gover, H.P. Freund, V.L. Granatstein, J.H. McAdoo, and C.M. Tang, "Basic Design Considerations for Free-Electron Lasers Driven by Electron Beams from rf Accelerators", in Infrared and Millimeter Waves, ed. K.J. Button (Academic, Orlando, 1984), vol. 11, chap. 8.

15. R.L. Sheffield, D.C. Nguyen, J.C. Goldstein, N.A. Ebrahim, C.M. Fortgang, and J.M. Kinross-Wright, "Compact $1 \mathrm{~kW}$ infrared regenerative amplifier FEL", in FreeElectron Laser Challenges, P.G. O'Shea and H.E. Bennett, eds. Proc. SPIE, vol. 2988, pp. 28-37, 1997.

16. J.M.J. Madey, "Relationship between mean radiated energy, mean squared radiated energy and spontaneous power spectrum in a power series expansion of the equations of motion in a free-electron laser" Nuovo Cimento, vol. 50B, pp. 64-88, 1979. 
17. H.P. Freund and A.K. Ganguly, "Three-dimensional theory of the free-electron laser in the collective regime", Phys. Rev A, vol. 28, pp. 3438-3449, 1983.

18. L.H. Yu, S. Krinsky, and R.L. Gluckstern, "Calculation of universal scaling function for free-electron laser gain", Phys. Rev. Lett., vol. 64, pp. 3011-3014, 1990.

19. Y.H. Chin, K.J. Kim, and M. Xie, "Three-dimensional theory of the small-signal highgain free-electron laser including betatron oscillations", Phys. Rev. A vol. 46, pp. 6662-6683, 1992.

20. J.C. Slater, Microwave Electronics (Van Nostrand, New York, 1950).

21. F.G. Yee, J. Masud, T.C. Marshall, and S.P. Schlesinger, "Power and sideband studies of a Raman free-electron laser", Nucl. Instrum. Meth., vol. A259, pp. 104-106, 1987.

22. J. Masud, T.C. Marshall, S.P. Schlesinger, F.G. Yee, W.M. Fawley, E.T. Scharlemann, S.S. Yu, A.M. Sessler, and E.J. Sternbach, "Sideband control in a millimeter-wave free-electron laser" Phys. Rev. Lett., vol. 58, pp. 763-766, 1987.

23. J.C. Goldstein, B.E. Newnam, and R.W. Warren, "Sideband suppression by an intracavity optical filter in the Los Alamos free-electron laser oscillator" Nucl. Instrum. Meth., vol. A272, pp. 150-154, 1988.

24. E.T. Scharlemann, A.M. Sessler, and J.S. Wurtele, "Optical guiding in a free-electron laser" Phys. Rev. Lett., vol. 54, pp. 1925-1928, 1985.

25. G.T. Moore, "High gain and large diffraction regimes of the free-electron laser", Nucl. Instrum. Meth., vol. A250, pp. 381-388, 1986.

26. P. Sprangle, A. Ting, and C.M. Tang, "Analysis of radiation focusing and steering in the free-electron laser", Phys. Rev. A, vol. 36, pp. 2773-2781, 1987.

27. A. Doria, G.P. Gallerano, and A. Renieri, "Kinematic and dynamic properties of a waveguide free-electorn laser" Opt. Commun., vol. 80, pp. 417-424, 1991.

28. N. Piovella et al., "Analytical theory of short pulse fere-elecron laser oscillators", Phys. Rev. E, vol. 52, pp. 5470-5486, 1995.

29. C.A. Brau, Free-Electron Lasers (Academic Press, Boston, 1990). 
30. P. Luchini and H. Motz, Undulators and Free-Electron Lasers (Clarendon Press, Oxford, 1990).

31. G. Ramian, "The New UCSB Free-Electron Lasers," Nucl. Instrum. Meth., A318 (1992), pp. 225-229.

32. B. C. Lee, S. K. Kim, Y. U. Jeong, S. O. Cho, B. H. Cha, and J. Lee, "First Lasing of the KAERI Millimeter-Wave Free Electron Laser," Nucl. Instrum. Meth., A375 (1996), pp. 28-31.

33. M. Caplan, M. Valentini, C. Van Der Geer, W. Urbanus, "Design and Characterization of the DC Acceleration and Transport System Required for the 1 MW Free Electron Maser Experiment," Nucl. Instrum. Meth., A375 (1995) pp. 91-94.

34. A. Abramovich, A. Arensburg, D. Chairman, A. Eichenbaum, M. Cohen, M. Draznin, A. Gover, H. Kleinman, Y. Pinhasi, J. S. Sokolowski and I. M. Yakover, "Anticipated Performance Parameters of the TAU Tandem FEL," Nucl. Instrum. Meth. A373 (1997) pp. II-41-44.

35. G. Ramian, J. Kaminski, S. J. Allen, "First Lasing of the UCSB $30 \mu \mathrm{m}$ Free-Electron Laser,” Nucl. Instrum. Meth., A393 (1997) pp. 220-224.

36. C. Brau, "The Vanderbilt University Free-Electron Laser Center," Nucl. Instrum. Meth., A318 (1992) pp. 38-41.

37. M. D. Shinn, "The Jefferson Laboratory IR Demo Project." Proceedings of SPIE Conference (FEL Challenges), February 13-14, 1997, San Diego, CA, 2988 (1997) pp. 170-175.

38. J. L. Adamski, D. H. Dowell, T. D. Hayward, C. G. Parazzoli, A. M. Vetter, "Proposed visible FEL facility at Boeing," Nucl. Instrum. Meth. A375 (1996) pp. 288-291.

39. R. L. Sheffield, D. C. Nguyen, J. C. Goldstein, N. A. Ebrahim, C. M. Fortgang and J. M. Kinross-Wright, "A Compact 1 KW Infrared Regenerative Amplifier FEL," 
Proceedings of SPIE Conference (FEL Challenges), February 13-14, 1997, San Diego, CA, 2988 (1997) pp. 28-37.

40. D. H. Dowell, S. Z. Bethel, K. D. Friddell, "Results from the high average power laser experiment photocathode injector test,” Nucl. Instrum. Meth. A356 (1995) pp. 167176.

41. T. Tomimasu, S. Okuma, K. Wakita, T. Takii, E. Oshita, A. Koga, S. Nishihara, A. Nagai, H. Tongu, K. Wakisaka, T. Mitsuyu, S. Kuribayashi, K. Nishi, H. Ohyama, T. Suzuki, E. Nishimura, K. Awazu, S. Ogino and M. Yasumoto, "Recent Status of Five FEL Facilities and Application Research at FELI," Free Electron Laser and Its Applications in Asia, pp. 65-74, 1997.

42. G. R. Neil, J. A. Edighoffer, and S. W. Fornaca, "Superconducting Linac FEL Technology in Free Electron Lasers," Proceedings of the Beijing FEL Seminar 11-23 August, 1988, Beijing China, Chen Jiaer, Xie Jialin, Du Xiangwan and Zhao Kui Editors, Published by World Scientific, Singapore, 1988.

43. D. A. G. Deacon, L. R. Elias, J. M. J. Madey, G. J. Ramian, H. A. Schwettman, and T. I. Smith, "First Operation of a Free-Electron Laser," Phys. Rev. Lett. 38 (1977) pp. 892-894.

44. J. A. Edighoffer, G. R. Neil, C. E. Hess, T. I. Smith, S. W. Fornaca, and H. A. Schwettman, "Variable-Wiggler Free-Electron-Laser Oscillation," Phys. Rev. Lett. 52 (1984) pp. 344-347.

45. J. A. Edighoffer, G. R. Neil, S. Fornaca, and H. R. Thompson, Jr., and T. I. Smith, H. A. Schwettman, C. E. Hess, J. Frisch, and R. Rohatgi, "Visible free-electron-laser oscillator (constant and tapered wiggler)," Appl. Phys. Lett. 52 (1988) pp. 15691570.

46. M. E. Couprie, "Storage Rings FELs," Nucl. Instrum. Meth., A393 (1997) pp. 1317. 
47. G. N. Kulipanov, V. N. Litvinenko, I. V. Pinaev, V. M. Popik, A. N. Skrinsky, A. S. Sokolov and N. A. Vinokurov, "The VEPP-3 Storage-Ring Optical Klystron: Lasing in the Visible and Ultraviolet Regions," Nucl. Instrum. Meth. A296 (1990) pp. $1-3$.

48. G. Dattoli and A. Renieri, "Storage ring operation of the free-electorn laser: the oscillator", Nuovo Cim., vol. 59B, pp. 1-39, 1980.

49. V. Litvinenko, Y. Wu, B. Burnham, S. H. Park, M. Emamian, J. Faircloth, S. Goetz, N. Hower, J. M. J. Madey, J. Meyer, P. Morcombe, O. Oakeley, J. Patterson, R. Sachtschale, G. Swift, and P. Wang, "First UV/Visible Lasing with the OK-4/Duke Storage Ring FEL: Design and Initial Performance," Proceedings of SPIE Conference (FEL Challenges), February 13-14, 1997, San Jose, CA, 2988 (1997) pp.188-199.

50. N. A. Vinokurov,I. B. Drobyazko, G. N. Kulipanov, V. N. Litvinenko, I. V. Pinayev, "Lasing in visible and ultraviolet regions in an optical klystron installed on the VEPP-3 storage ring,” Rev. Sci. Instr., vol. 60, pp. 1435-1438, 1989.

51. S. Penner, R. Ayres, R. Cutler, P. Debenham, B. C. Johnson, E. Lindstrom, D. Mohr, J. Rose, M. Wilson, P. Sprangle and C. M. Tang, "The NBS/NRL Free Electron Laser Facility,” Nucl. Instrum. Meth., vol. A272, pp. 73-80, 1988.

52. T. Hara, M. E. Couprie, A. Delboulbé, P. Troussel, D. Gontier, and M. Billardon, "Observations of the Super-ACO FEL Micropulse with a Streak Camera," Nucl. Instrum. Meth., vol. A341, pp. 21-23, 1994.

53. I. B. Drobyazko, G. N. Kulipanov, V. N. Litvinenko, I. V. Pinayev, V. M. Popik, I. G. Silvestrov, A. N. Skrinsky, A. S. Sokolov, and N. A. Vinokurov, "Lasing in Visible and Ultraviolet Regions in an Optical Klystron Installed on the VEPP-3 Storage Ring,” Nucl. Instrum. Meth., vol. A282, pp. 424-430, 1989. 
54. S. Takano, H. Hama, and G. Isoyama, "Lasing of a Free Electron Laser in the Visible on the UVSOR Storage Ring," Nucl. Instrum. Meth., vol. A331, pp. 20-26, 1993.

55. T. Yamazaki, K. Yamada, S. Sugiyama, H. Ohgaki, N. Sei, T. Mikado, T. Noguchi, M. Chiwaki, R. Suzuki, M. Kawai, M. Yokoyama, K. Owaki, S. Hamada, K. Aizawa, Y. Oku, A. Iwaa and M. Yoshiwa, "First Lasing of the NIJI-IV StorageRing Free Electron Laser,” Nucl. Instrum. Meth., vol. A331, pp. 27-33, 1993.

56. P. G. O'Shea, S. C. Bender, D. A. Byrd, J. W. Early, D. W. Feldman, C. M. Fortgang, J. C. Goldstein, B. E. Newnam, R. L. Sheffield, R. W. Warren and T. J. Zaugg, "Demonstration of Ultraviolet Lasing with a Low Energy Electron Beam," Nucl. Instrum. Meth., vol. A341, pp. 7-11, 1994.

57. N. G. Gavrilov, e. I. Gorniker, D. A. Kayran, G. N. Kulipanov, I. V. Kuptsov, G. Y. Kurkin, E. I. Kolobanov, Y. I. Levashov, A. D. Oreshkov, S. P. Petrov, V. M. Petrov, I. V. Pinayev, V. M. Popik, t. V. Salikova, I. K. Sedlyarov, T. V. Shaftan, A. N. Skrinsky, N. A. Vinokurov, P. D. Vobly, and E. M. Zakutov, "Status of the Novosibirsk High Power Free Electron Laser Project," Proceedings of SPIE Conference (FEL Challenges), February 13-14, 1997, San Jose, CA, SPIE 2988 (1997) pp. 185-187.

58. B. E. Carlsten, "New Photoelectric Injector Design for the Los Alamos National Laboratory XUV FEL Accelerator,” Nucl. Instrum. Meth., vol. A285, pp. 313-319, 1989.

59. D. T. Palmer, X. J. Want, R. H. Miller, M. Babzien, I. Ben-Zvi, C. Pellegrini, J. Sheehan, J. Skaritka, T. Srinivasan-Rao, H. Winick, M. Woodle, and V. Yakimenko, "Commissioning Results of the BNL/SLAC/UCLA Symmetrized 1.6 Cell S-Band Emittance Compensated Photoinjector," Proceedings of SPIE Conference (FEL Challenges), February 13-14, 1997, San Jose, CA, SPIE 2988 (1997) pp. 78-89. 
60. J. F. Schmerge, D. A. Reis, M. Hernandez, D. D. Meyerhofer, R. H. Miller, D. T. Palmer, J. N. Weaver, H. Winick, and D. Yeremian, "SLAC RF Photocathode Gun Test Facility," Proceedings of SPIE Conference (FEL Challenges), February 13-14, 1997, San Jose, CA, SPIE 2988 (1997) pp. 90-96.

61. S. H. Kong, J. Kinross-Wright, D. C. Nguyen, R. L. Sheffield, "Photocathodes for Free Electron Lasers,” Nucl. Instrum. Meth., vol. A358, pp. 272-275, 1995.

62. P. Michelato, "Photocathodes for RF Photoinjectors," Nucl. Instrum. Meth., vol. A393, pp. 455-459, 1997.

63. C. K. Sinclair, "A $500 \mathrm{kV}$ Photoemission Electron Gun for the CEBAF FEL," Nucl. Instrum. Meth., vol. A318, pp. 410-414, 1992.

64. A. Michalke, H. Piel, C. K. Sinclair, P. Michelato, C. Pagani, L. Serafini, M. Peiniger, "Photocathodes inside Superconducting Cavities," 5th Workshop on RF Superconductivity, Hamburg Germany, (1991) pp. 734-742.

65. A. Buchner, H. Buttig, F. Gabriel, P. Gippner, E. Grosse, D. Janssen, U. Nething, F. Pobeel, H. Prade, R. Schlenk, K.-D. Schilling, W. Seidel, P. Vom Stein, J. Voightlander, M. Wenzel, W. Wendler, A. Wolf, R. Zahn, "The Project of a FarInfrared FEL at the Rossendorf Radiation Source ELBE,' at the 19th International Free Electron Laser Conference, Beijing, 1997, to be published in Nucl. Instrum. Meth. A.

66. S. V. Benson, B. Richman, and L. Vintro, "A Review of the Stanford Mark III Infrared FEL Program,” Nucl. Instrum. Meth., vol. A296, pp. 110-114, 1990.

67. G. Edwards and N. H. Tolk, "Vanderbilt University FEL Center for Biomedical and Materials Research,” Nucl. Instrum. Meth., vol. A272, pp. 37-39, 1988.

68. J. Xie, J. Zhuang, Y. Huang, Y. Li, S. Lin, R. Ying, Y. Zhong, L. Zhang, G. Wu, Y. Zhang, C. Chao, L. Li, E. Fu, J. Su, Y. Wang and G. Wang, "The Saturation of the Beijing FEL,” Nucl. Instrum. Meth., vol. A358, pp. 256-259, 1995. 
69. G. A. Deis A. R. Harvey, C. D. Parkison, D. Prosnitz, J. Rego, E. T. Scharlemann and K. Halbach, "A Long Electromagnetic Wiggler for the PALADIN Free-Electron Laser Experiments," IEEE Trans. Magnetics, vol. 24, pp. 1090-1097, 1988.

70. T. I Smith, H. A. Schwettmann, K. W. Berryman, R. L. Swent, "Facilities at the Stanford Picosecond FEL Center," Proc. SPIE 1854, (1993) pp. 23-33

71. Y. Wu, V. N. Litvinenko, J. M. J. Madey, "Lattice and Dynamic Aperture of the Duke FEL Storage Ring,” Proc. 1995 PAC Dallas TX, May 1-5 (1995) pp. 213-220

72. K. Halbach, "Design of Permanent Multipole Magnets with Oriented Rare Earth Cobalt Material,” Nucl. Instrum. Meth., vol. 169, pp. 1-10, 1980.

73. C. J. Elliot, and B. D. McVey, "Undulator magnets for Synchrotron Radiation and Free Electron Lasers," Symp. in the Adriatico Conf. Series, Trieste, Italy 1987 (World Scientific Teaneck, NJ 1988) pp. 142-148.

74. C. M. Fortgang and R.W. Warren, "Measurement and Correction of Magnetic Fields in Pulsed Slotted-Tube Microwigglers," Nucl. Instrum. Meth., vol. A341, pp. 436439, 1994.

75. S. C. Gottschalk, A. L. Pindroh, D. C. Quimby, K. E. Robinson and J. M. Slater, "Enhanced FEL Performance from Superconducting Undulators," Nucl. Instrum. Meth., vol. A304, pp. 732-737, 1991.

76. R. Stoner and G. Bekefi, "A 70-Period High-Precision Microwiggler for Free Electron Lasers,” IEEE J. Quantum Electron., vol. QE-31, pp. 1158-1165, 1995.

77. G. Ingold, I. Ben-Zvi, L. Solomon and M. Woodle., "Fabrication of a High-Field Short-Period Superconducting Undulator," Nucl. Instrum. Meth., vol. A375, pp. 451-455, 1996.

78. J. Pfluger, D. Koster, and T. Teichmann, “The Undulator Prototype Structure for the FEL at the TTF," Proc. 19th Int'l FEL Conf., Beijing, August 1997, to be published Nucl. Instrum. Meth. A. 
79. R. Tatchyn, R. Boyce, K. Halbach, H.-D. Nuhn, J. Seeman, H. Winick, “ Design Considerations for a 60 Meter Pure Permanent Magnet Undulator for the SLAC Linac Coherent Light Source (LCLS)," Proc. IEEE PAC95 Dallas TX May 1-5 1995, pp. $1608-1610$

80. C. M. Fortgang, “ A Pure Permanent Magnet - Two Plane Focusing - Tapered Wiggler for a High Average Power FEL,” Nucl. Instrum. Meth., vol. A393, pp. 385388, 1997.

81. R. Tatchyn, "Selected Applications of Planar Permanent Magnet Multipoles in FEL Insertion Device Design,” Nucl. Instrum. Meth., vol. A341, pp. 449-453, 1994.

82. A. A. Varfolomeev, V. V. Gubankov, A. H. Hairetdinov, S. N. Ivanchenkov, A. S. Khlebnikov, N. S. Osmanov, and S. V. Tolmachev, "Mock-up of the Focusing Undulator for the SLAC X-ray FEL Project,” Nucl. Instrum. Meth., vol. A358, pp. 70-71, 1995.

83. G. Dattoli, T. Hermsen, A Renieri, A. Torre and J. C. Gallardo, "Lethargy of Laser Oscillations and supermodes in free-electron lasers I.," Phys. Rev. A, vol. 37, pp. 4326-4333, 1988; and G. Dattoli, T. Hermsen, L. Mezi, A Renieri, and A. Torre, "Lethargy of Laser Oscillations and supermodes in free-electron lasers II.," Phys. Rev. A, vol. 37, pp. 4334-4339, 1988.

84. M. E. Couprie, D. Garzella and M. Billardon, "Optical Cavities for UV Free Electron Lasers,” Nucl. Instrum. Meth., vol. A358, pp. 382-386, 1995.

85. H. Hama, K. Kimura, M. Hosaka, J. Yamazaki and T. Kinoshita, "UV-FEL Oscillation Using a Helical Optical Klystron,", in FEL Applications in Asia, T. Tomimasu, E. Nishimika, T. Mitsuyu ed., Ionics Publishing, Tokyo.

86. K. Yamada, T. Yamazaki, N. Sei, R. Suzuki, T. Ohdaira, T. Shimizu, M. Kawai, M. Yokoyama, T. Mikado, T. Noguchi, S. Sugiyama and H. Ohgaki, "Saturation of Cavity-Mirror Degradation in the UV FEL," Nucl. Instrum. Meth., vol. A393, pp. 44-49, 1997. 
87. W. P. Leemans, M. Xie, J. A. Edighoffer, E. Wallace, K.-J. Kim and S. Chattopadhyay, "Experiment and Simulation of Hole-Coupled Resonator Modes with a CW HeNe Laser,” Nucl. Instrum. Meth., vol. A331, pp. 630-635, 1993.

88. G. R. Neil, S. V. Benson, M. D. Shinn, P. C. Davidson and P. K. Kloeppel, “Optical Modeling of the Jefferson Laboratory IR Demo FEL," Proceedings of SPIE Conference (Modeling and Simulation of Higher-Power Laser Systems IV), February 12-13, 1997, San Jose, CA, SPIE 2989, (1997) pp. 160-171.

89. C.-C. Shih, and S.-M. Shih, "Advanced Free Electron Laser Resonator," Nucl. Instrum. Meth., vol. A304, pp. 788-791, 1991.

90. N. A. Vinokurov, A. A. Zholents, W. M. Fawley, and K.-J. Kim, “Critical Issues for High-Power FEL Based on Microtron Recuperator/Electron Out-Coupling Scheme," Proceedings of SPIE Conference (FEL Challenges), February 13-14, 1997, San Diego, CA, SPIE 2988 (1997) pp. 78-89.

91. W. B. Colson, "Short Wavelength Free Electron Lasers in 1996," Nucl. Instrum. Meth., vol. A393, pp. 6-8, 1997.

92. H. P. Freund and V. P. Granatstein, "Long Wavelength Free Electron Lasers in 1996,” Nucl. Instrum. Meth., vol. A393, pp. 9-12, 1997.

93. W. M. Fawley, "Output Characteristics of SASE-Driven Short Wavelength FEL's," Proceedings of SPIE Conference (FEL Challenges), February 13-14, 1997, San Diego, CA, SPIE 2988 (1997) pp. 98-107.

94. W. Brefeld, B. Faatz, Y. M. Nikitina, J. Pfluger, P. Pierini, J. Robbach, E. L. Saldin, E. A. Schneidmiller and M. V. Yurkov, "Parameter Study of the VUV FEL at the TESLA Test Facility,” Nucl. Instrum. Meth., vol. A375, pp. 295-298, 1996.

95. R. Li, C. L. Bohn, J. J. Bisognano, "Analysis on the Steady State Coherent Radiation with Strong Shielding," Proc. 1997 Particle Accelerator Conf., Vancouver, B.C., Canada, May 11-15, 1997 and R. Li, C. L. Bohn, J.J. Bisognano, "Shielded Transient Self-Interaction of a Bunch Entering a Circle from a Straight 
Path,” Proc. 1997 Particle Accelerator Conf., Vancouver, B.C., Canada, May 11-15, 1997.

96. M. Cornacchia, "Performance and Design Concepts of a Free Electron Laser Operating in the X-ray Region,” Proceedings of SPIE Conference (FEL Challenges), February 13-14, 1997, San Diego, CA, SPIE 2988 (1997) pp. 2-13.

97. R. Tatchyn, "Prospects for the Stimulation, Control and Study of High-Gain LinacDriven X-ray Free Electron Laser (FEL) Interactions Using High-Power IR/Visible/UV Laser,” Proceedings of SPIE Conference (FEL Challenges), February 13-14, 1997, San Diego, CA, SPIE 2988 (1997) pp. 112-126.

98. J. Rossbach, "A VUV Free Electron Laser at the TESLA Test Facility at DESY," Nucl. Instrum. Meth., vol. A375, pp. 269-273, 1996.

99. T. J. Orzechowski, B. R. Anderson, J. C. Clark, W. M. Fawley, A. C. Paul, D. Prosnitz, E. T. Scharlemann, S. M. Yarema, D. B. Hopkins, A. M. Sessler, J. S. Wurtele, "High Efficiency Extraction of Microwave Radiation from a Tapered Wiggler Free Electron Laser,” Phys. Rev. Lett., vol. 57, pp. 2172-2175, 1986.

100. D. A. Kirkpatrick, G. Bekefi, A. C. DiRienzo, H.P. Freund, and A.K. Ganguly, “A millimeter and submillimeter wavelength free-electron laser," Phys. Fluids, vol. B1, pp. 1511-1518, 1989.

101. D. C. Nguyen, R. L. Shenffield, C. M. Fortgang, J. C. Goldstein, J. M. KinrossWright and N. A. Ebrahim, "Demonstration of High-Gain SASE and High-Power Regenerative Amplifier FEL," Proc. 19th Int'1 FEL Conf., Beijing, (1997) to be published in Nucl. Instrum. Meth. A.

102. P. G. O'Shea, C. P. Neuman, J. M. J. Madey, and H. P. Freund, "Short Wavelength SASE Using the PALADIN Wiggler," Nucl. Instrum. Meth., vol. A393, pp. 129$132,1997$.

103. R. Mabe, Limit cycle Behavior in Free Electron Lasers, Master Thesis in Physics, Naval Postgraduate School (Fall, 1995). 
104. Sang June Hahn and Jae Koo Lee, "Nonlinear short-pulse propagation in a freeelectron laser," Phys. Rev. E, vol. 48, pp. 2162-2171, 1993.

105. Donald W. Feldman, Roger W. Warren, Bruce E. Carlsten, William E. Stein, Alexander H. Lumpkin, S. C. Bender, G. Spalek, Jerry M. Watson, L. M. Young, John S. Fraser, John C. Goldstein, Harunori Takeda, Tai-sen F. Wang, Kwok-Chi Dominic Chan, B. D. McVey, Brian E. Newnam, R. A. Lohsen, Renee B. Feldman, Richard K. Cooper, W. Joel D. Johnson, and Charles A. Brau, "Recent Results of the Los Alamos Free-Electron Laser,” IEEE J. Quantum Electron., vol. QE-23. pp. 1476-1488. 1987.

106. T. I. Smith, H. A. Schwettman, R. Rohatgi, Y. Lapierre, and J. Edighoffer, "Development of the SCA/FEL for use in biomedical and materials science experiments," Nucl. Instrum. Meth., vol. A259, pp. 1-7, 1987.

107. D. Oepts, R. J. Bakker, D. A. Jaroszynski, A. F. G. van der Meer, P. W. van Amersfoort, "Narrow-band Operation of FELIX," Nucl. Instrum. Meth., vol. A341, p. ABS28, 1994.

108. D. A. Jaroszynski, R. Prazeres, F. Glotin, O. Marcouillé, J. M. Ortega, D. Oepts, A. F. G. van der Meer, G. M. H. Knippels, W. W. van Amersfoort, "Step-tapered Operation of the FEL: Efficiency Enhancement and Two-Colour Operation," Nucl. Instrum. Meth., vol. A375, pp. 647-650, 1996.

109. S.V. Benson and J.M.J. Madey, "Demonstration of harmonic lasing in a freeelectron laser," Phys. Rev. A, vol. 39, pp. 1579-1581, 1989.

110. M. Marsi, M. E. Couprie, L. Nahon, D. Garzella, R. Bakker, A. Delboulbé, D. Nutarelli, R. Roux, B. Visentin, C. Grupp, G. Indlekofer, G. Panaccione, A. TalebIbrahimi, M. Billardon, "Two Color Experiments Combining Free Electron Laser and Synchrotron Radiation,” Nucl. Instrum. Meth., vol. A393, pp. 548-551, 1997. 
111. M. Curtin, A. Bhowmik, J. Brown, W. McMullin, P. Metty, S. V. Benson, and J. M. J. Madey, "Initial Results of Operating the Rocketdyne Undulator in a Tapered Configuration,” Nucl. Instrum. Meth., vol. A296, pp. 69-74, 1990.

112. F. V. Hartemann, T. S. Chu, A. L. Troha, J. R. Van Meter, G. P. Le Sage, C. V. Bennett, V. V. Dinh, B. H. Kolner, J. P. Heritage, H. Baldis and N.C. Luhmann, "Compton Backscattering Focused X-ray Source for Advanced Biomedical Applications," Proceedings of SPIE Conference (FEL Challenges), February 13-14, 1997, San Jose, CA, SPIE 2988 (1997) pp. 52-63.

113. V. Litvinenko, Y. Wu, B. Burnham, S. H. Park, M. Emamian, J. Faircloth, S. Goetz, N. Hower, J. M. J. Madey, J. Meyer, P. Morcombe, O. Oakeley, J. Patterson, R. Sachtschale, G. Swift, and P. Wang, "First UV/Visible Lasing with the OK-4/Duke Storage Ring FEL: Design and Initial Performance," Proceedings of SPIE Conference (FEL Challenges), February 13-14, 1997, San Jose, CA, SPIE 2988 (1997) pp. 188-194.

114. H. R. Weller, "Nuclear Physics Studies Using a Free-Electron Laser GeneratedBeam,” Nucl. Instrum. Meth., vol. A393 pp. II-121-124, 1997.

115. M. Hosaka, H. Hama, K. Kimura, J. Yamazaki and T. Kinoshita, "High Energy Photons from Intracavity Compton Backscattering in the UVSOR Storage Ring," FEL and Its Application in Asia, T. Tomimasu, E. Nishimika, T. Mitsuyu ed., Ionics Publishing, Tokyo, (1997) pp. 25-30.

116. F. Ciocci, G. Dattoli, L. Giannessi, P. L. Ottaviani, M. Quattromini, and M. Carpanese, "Compton Backscattering Induced by FEL Photons: A General Treatment," Nucl. Instrum. Meth., vol. A393, pp. 536-539, 1997.

117. M. Hosaka, H. Hama, K. Kimura, J. Yamazaki, T. Kinoshita, "Observation of Intracavity Compton Backscattering of the UVSOR Free Electron Laser," Nucl. Instrum. Meth., vol. A393, pp. 525-529, 1997. 
118. V. Litvinenko and J. M.J. Madey, "Intense Compton $\gamma$-ray Source from the Duke Storage Ring FEL,” Nucl. Instrum. Meth., vol. A359, pp. 580-583, 1996.

119. F. Glotin, J.-M. Ortega, R. Prazeres, G. Devanz, O. Marcouillé, “Tunable X-rays Generation in a Free-Electron Laser by Intracavity Compton Backscattering," Nucl. Instrum. Meth., vol. A393, pp. 519-524, 1997.

120. Free Electron Lasers and Other Advanced Sources of Light, report of the Committee on Free Electron Lasers, National Research Council, National Academy Press, Washington, DC 1994.

121. H. A. Schwettman, "Challenges at FEL Facilities: The Stanford Picosecond FEL Center," Nucl. Instrum. Meth., vol. A375, pp. 632-638, 1996.

122. S. J. Allen, K. Craig, B. Galdrikian, J. N. Heyman, J. P. Kaminski, J. S. Scott, M. S. Sherwin, K. Unterrainer, M. Wanke, K. Campman, P. F. Hopkins, A. C. Gossard, D. H. Chow, M. Lui, and T. K. Liu, "Materials Science in the Far-IR with Electrostatic Based FELs,” Nucl. Instrum. Meth., vol. A358, pp. 536-539, 1995.

123. G. M. Lankhuijzen and L. D. Noordam, "Far-Infrared Streak Camera," Nucl. Instrum. Meth., vol. A375, pp. 651-653, 1996.

124. A. Peremans, A. Tadjeddine, P. Guyot-Sionnest, R. Prazeres, F. Glotin, D. Jaroszynski, J. M. Berset, and J.-M. Ortega, “Adsorbate Vibrational Spectroscopy by IR-Visible Sum-Frequency Generation Using CLIO-FEL: $\mathrm{CO}$ from $\mathrm{CH}_{3} \mathrm{OH}$ Electrochemical Deposition on Pt," Nucl. Instrum. Meth., vol. A341, pp. 146-151, 1994.

125. A. Peremans, A. Tadjeddine, W. Q. Zheng, P. Guyot-Sionnest, P. Remy, G. Ryschenkow, M. Buck, Y. Caudano L.-M. Yu, P. A. Thiry, B. Bourguignon, H. Dubost, B. Draggnea, and S. Carrez, "Vibrational Spectroscopy at Interfaces by IRVIS Sum-Frequency Generation Using CLIO FEL," Nucl. Instrum. Meth., vol. A375, pp. 657-661, 1996. 
126. A. K. Petrov, E. N. Chesnokov, S. R. Gorelik, Y. N. Molin, K. D. Straub, E. Szarmes and J. M. J. Madey, "Isotope Selective Multiphoton Dissociation of Formic Acid and Nitromethane by a Free Electron Laser," FEL and Its Applications in Asia, T. Tomimasu, E. Nishimika, T. Mitsuyu ed., Ionics Publishing, Tokyo. (1997) pp. 245-252.

127. H. C. Chui, G. L. Woods, M. M. Fejer, E. L. Martinet, and J. S. Harris, Jr., "Tunable mid-infrared generation by difference frequency mixing of diode laser wavelengths in intersubband InGaAs/AlAs quantum wells," Appl. Phys. Lett. 66 (1995) 265-267.

128. A. Tokmakoff and M. D. Fayer, "Homogeneous Vibrational Dynamics and Inhomogeneous Broadening in Glass-Forming Liquids: Infrared Photon Echo Experiments from Room Temperature to 10 K”, J. Chem. Phys., vol. 103, pp. 2810$2817,1995$.

129. Yoshihito Maeda, Kenji Umezawa, Shigehiro Ohwaki, and Yoshikazu Hayashi, "Structural Relaxation of Hydrogenated Amorphous Silicon Using Resonant Vibrational Excitation with Free Electron Lasers," FEL and Its Applications in Asia, T. Tomimasu, E. Nishimika, T. Mitsuyu ed., Ionics Publishing, Tokyo. (1997) pp. 307-314

130. T. Suzuki,. T. Mitsuyu, K. Nishi, H. Ohyama, T. Tomimasu, S. Noda, T. Asano, A. Saski, "Observation of ultrafast all-optical modulation based on intersubband transition in n-doped quantum wells by using a free electron laser", Appl. Phys. Lett., vol. 69, pp. 4136-4138, 1996.

131. S. Kuribayashi, T. Tomimasu, S. Kawanishi, and S. Arai, "Experimental Studies on Laser Isotope Separation of carbon and silicon by a Free Electron Laser,", FEL and Its Applications in Asia, T. Tomimasu, E. Nishimika, T. Mitsuyu ed., Ionics Publishing, Tokyo. (1997) pp. 263-270 
132. C. Brau, "Bright Beams and Small FELs," Proceedings of 19th Int'l FEL Conf. Beijing, August 1997 to be published Nucl. Instrum. Meth..

133. Seiji Ogino, Kunio Awazu, Takio Tomimasu, "Effect of free electron laser (FEL) irradiation on tooth dentine", Proc. SPIE Vol. 2922, pp. 184-192, 1996.

134. T. Tomimasu, S. Okuma, K. Wakita, T. Takii, E. Oshita, A. Koga, S. Nishihara, A. Nagai, H. Tongu, K. Wakisaka, T. Mitsuyu, S. Kuribayashi, K. Nishi, H. Ohyama, T. Suzuki, E. Nishimura, K. Awazu, S. Ogino, and M. Yasumoto, “ Recent Status of Five FEL Facilities and Applications Research at FELI," FEL and Its Applications in Asia, T. Tomimasu, E. Nishimika, T. Mitsuyu ed., Ionics Publishing, Tokyo. (1997) $65-74$

135. B. Jean, "Three Years of Biomedical FEL Use in Medicine and Surgery: How Far Have We Come?” Nucl. Instrum. Meth., vol. A393, pp. 540-543, 1997.

136. M. J. Kelley, “An FEL for the Polymer Processing Industries,” Proceedings of SPIE Conference (FEL Challenges), February 13-14, 1997, San Diego, CA, SPIE 2988 (1997) pp. 240-244.

137. M. E. Couprie, R. Bakker,D. Garzella, L. Nahn, M. Marsi, F. Mérola, T. Hara and M. Billardon, "Source Challenges Resulting from the First Applications of a UV Storage Ring FEL on Super-ACO,” Nucl. Instrum. Meth., vol. A375, pp. 639-643, 1996.

138. H. E. Bennet, "Progress in Establishing a Laser Power-Beaming Facility at China Lake, California," Proceedings of SPIE Conference (FEL Challenges), February 1314, 1997, San Diego, CA, SPIE 2988 (1997) pp. 232-237.

139. H. E. Bennet, "Laser Power Beaming: An Emerging Technology for Power Transmission and Propulsion in Space," Proceedings of SPIE Conference (FEL Challenges), February 13-14, 1997, San Diego, CA, SPIE 2988 (1997) pp. 245-256.

140. J. D. Cohen,C. W. Erkenbrecher, S. L. Haynie, M. J. Kelley, H. Kobsa, A. N. Roe, and M. H. Scholla, U.S. Patent No. 5,428,078, June 27, 1995. 
141. K.Yamada, T. Yamazaki, N. Sei, H. Ohgaki, T. Mikado, T. Noguchi, S. Sugiyama, R. Suzuki, T. Ohdaira, M. Kawai, and M. Yokoyama, "Challenge to Deep UV and VUV FEL in a Small-Scale Storage Ring," Proceedings of SPIE Conference (FEL Challenges), February 13-14, 1997, San Diego, CA, SPIE 2988 (1997) pp. 134-144.

142. K. J. Weeks, "Radiation Therapy Potential of Intense Backscattered Compton Photon Beams,” Nucl. Instrum. Meth., vol. A393, pp. 544-547, 1997. 


\section{FIGURE CAPTIONS}

Fig. 1 Progress in the development of vacuum electronic sources as measured by the growth in the product of the average power and the square of the frequency versus time.

Fig. 2 Schematic illustration of the interaction between the beam and the wiggler in an FEL with a planar wiggler.

Fig. 3 Schematic illustration of the resonance condition between the beam and the wave in an FEL with a helical wiggler [3].

Fig. 4 Graph of the spectral function for the spontaneous radiation from a cold beam.

Fig. 5 Schematic of the electron phase space evolution. The electron beam is initially (a) monoenergetic. During the linear phase of the interaction (b), the wave grows in amplitude and the separatrix expands. The bulk of the beam executes trapped orbits at saturation (c). The phase space distribution shown represents an electron beam in which the electrons still losing energy to the wave are balanced by those which are gaining energy from the wave [3].

Fig. 6 Schematic illustration of the evolution of the power versus axial distance showing the initial start-up region, the exponential growth phase, and saturation.

Fig. 7 Plot of the FEL spectral function for the gainin the initial start-up region.

Fig. 8 Schematic of an RF Linac-driven FEL oscillator.

Fig. 9 Schematic illustration of the proposed Linac Coherent Light Source at SLAC.

Fig. 10 The average brilliance predicted for the TTF-FEL and TESLA-FEL exceeds all third generation light sources by many orders of magnitude (figure courtesy $\mathrm{J}$. Rossbach., used by permission).

Fig. 11 Schematic illustration of the configuration of the high average power FEL under construction at Jefferson Laboratory. 


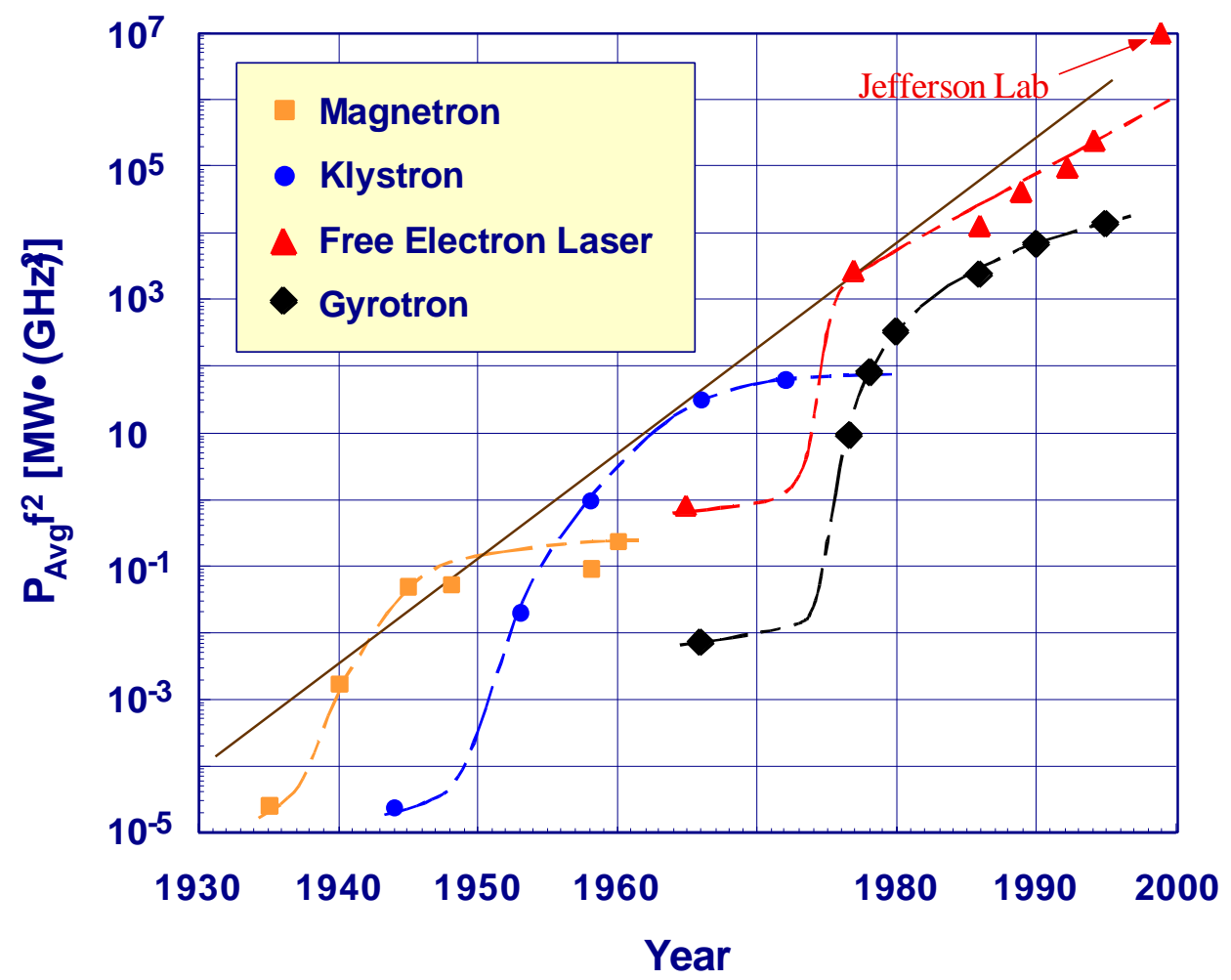


Fig. 1

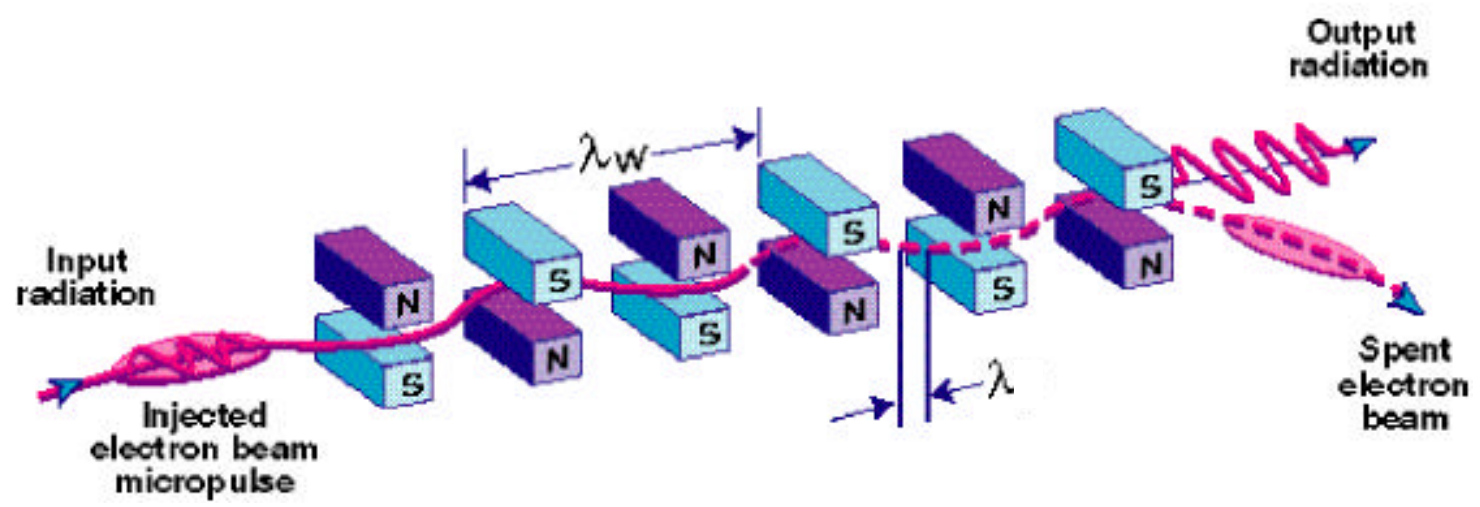


Fig. 2 


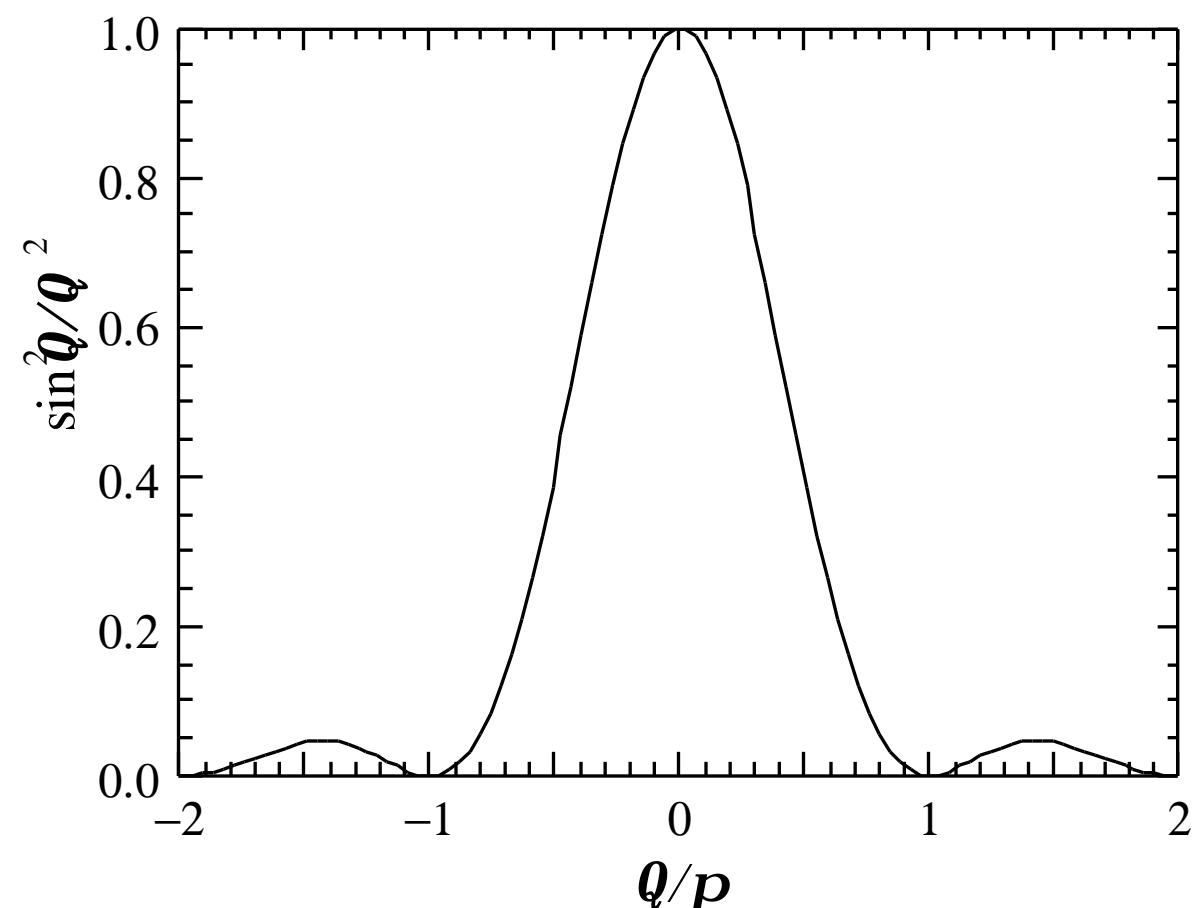


Fig. 4 

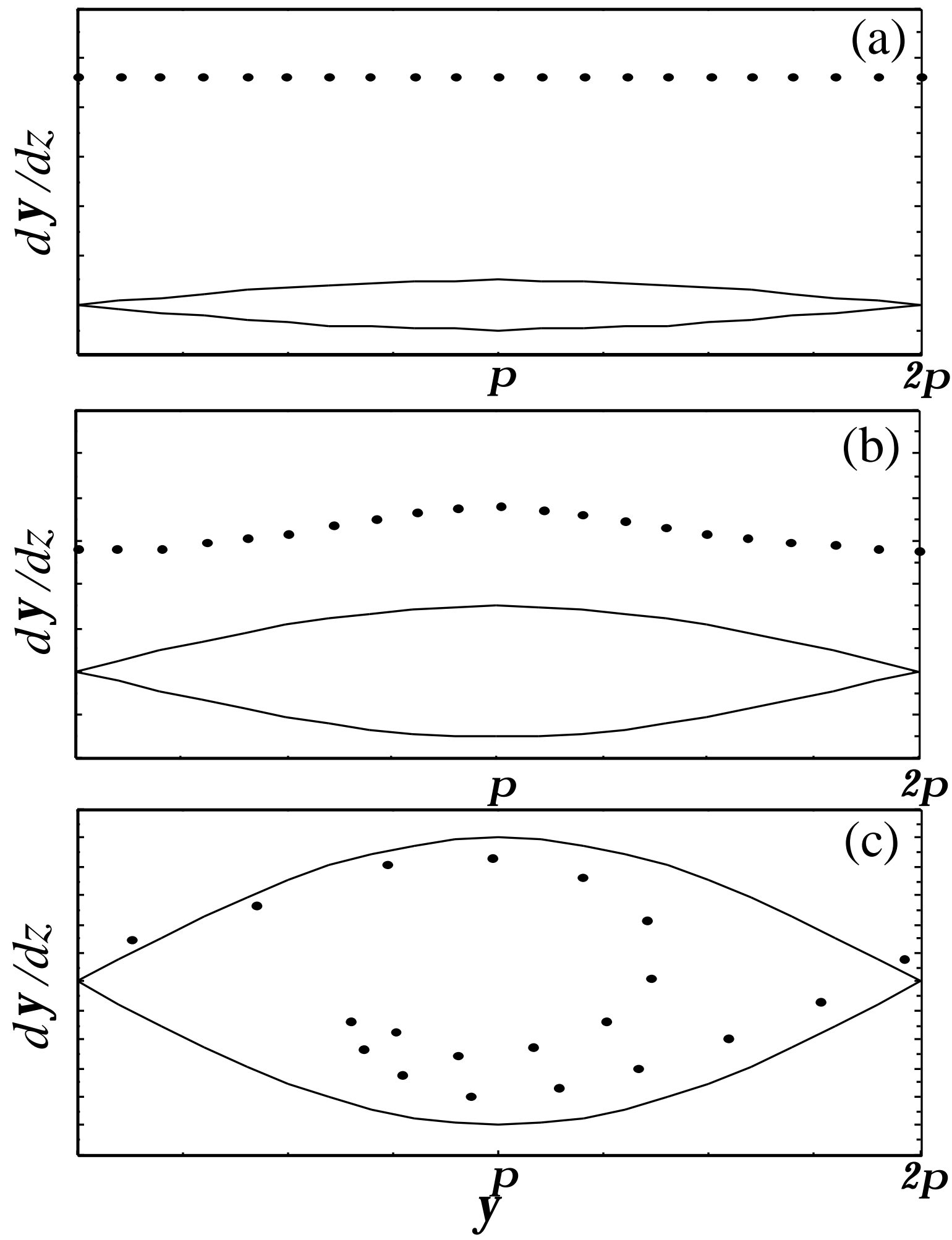

Fig. 5 


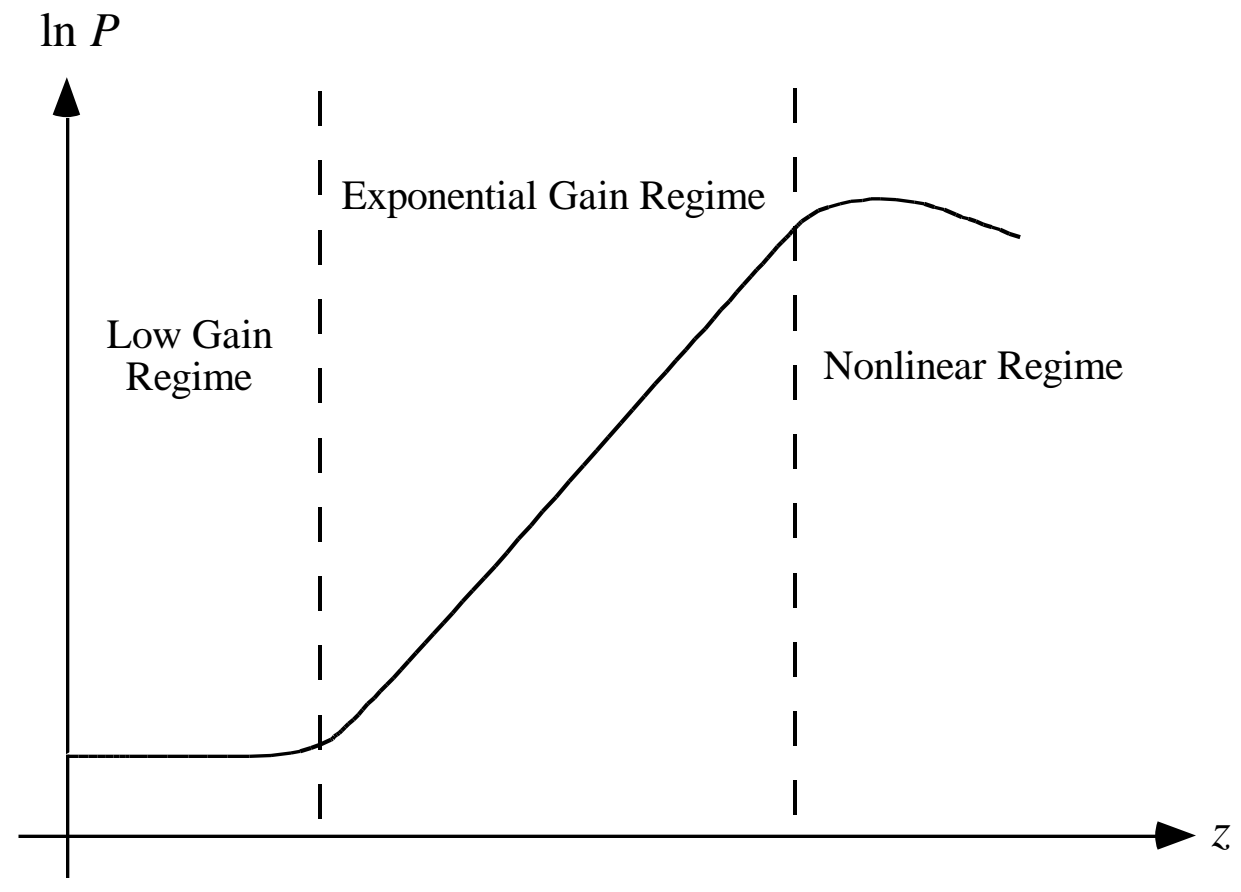


Fig. 6

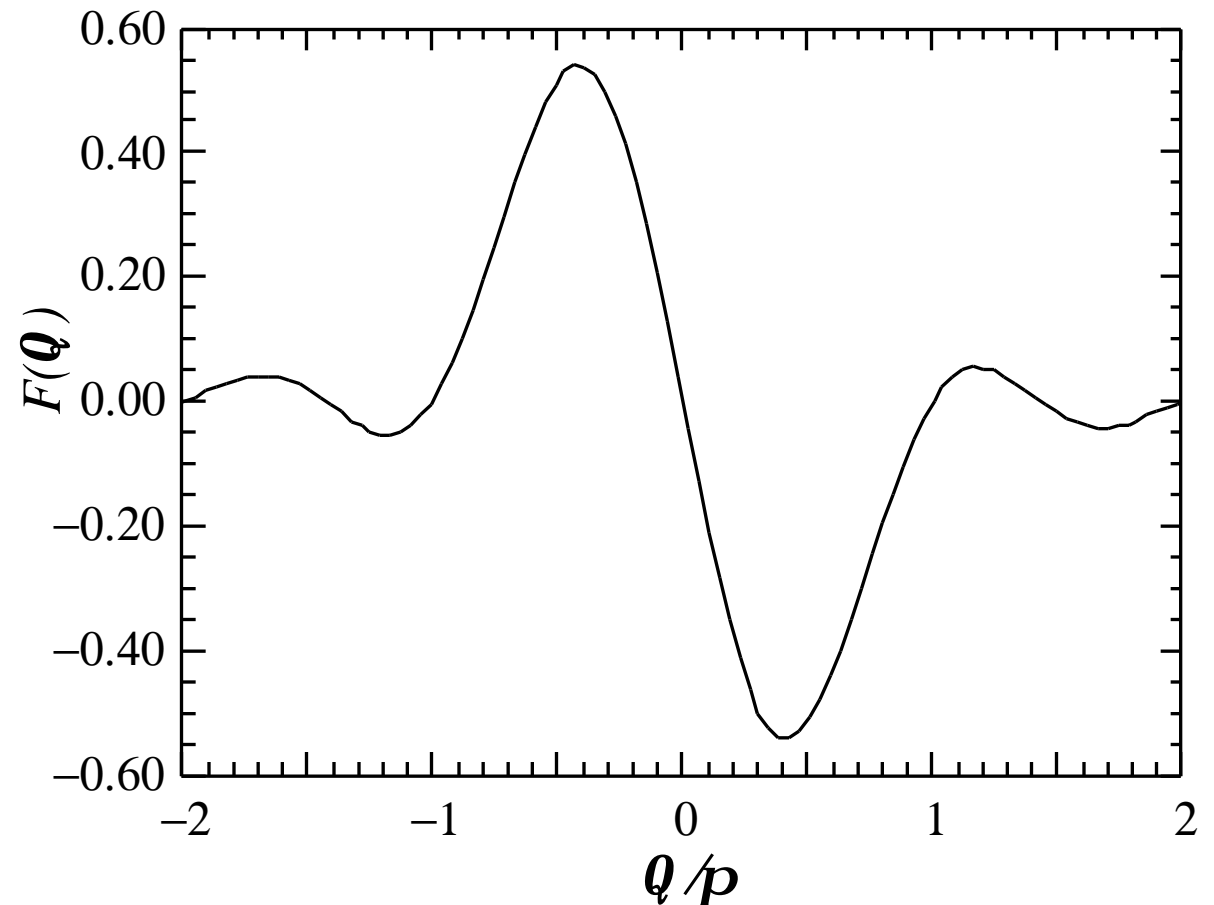


Fig. 7

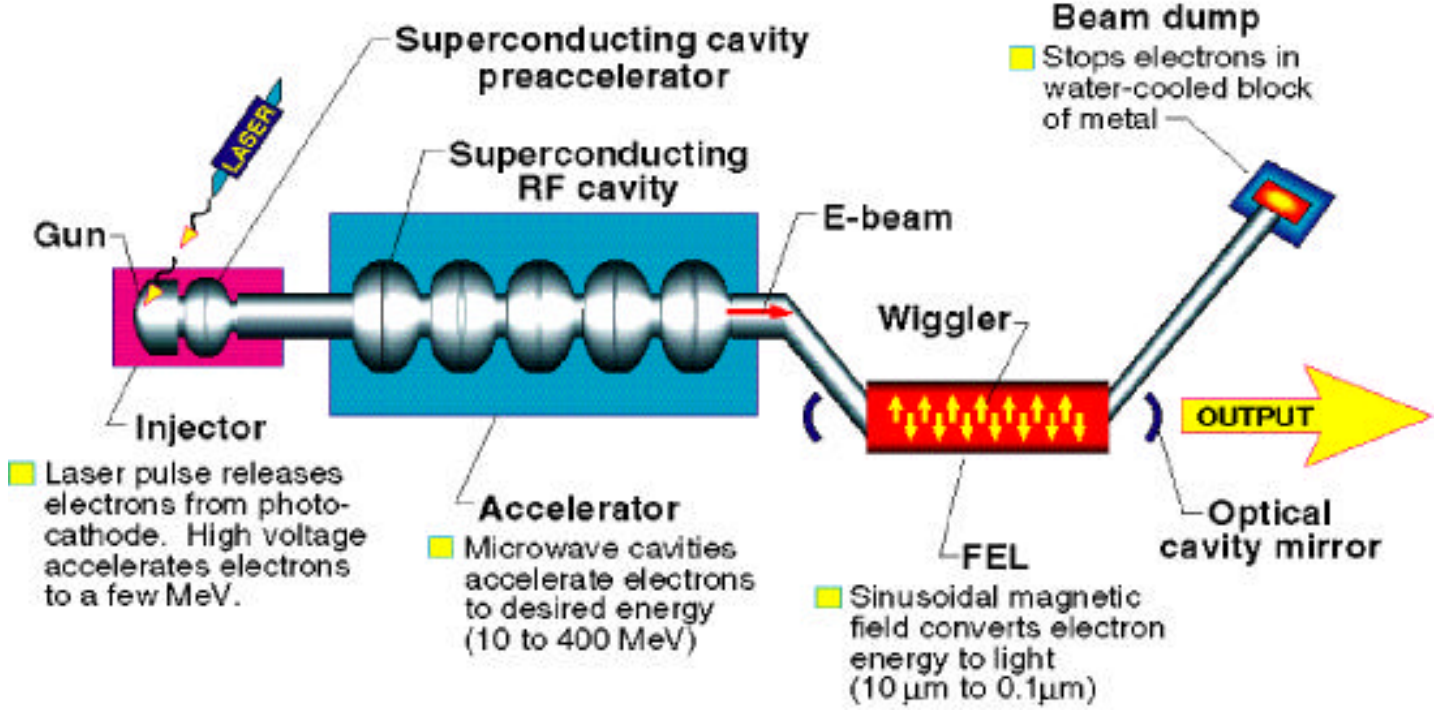

\section{Beam dump}

Stops electrons in water-cooled block 
Fig. 8

\section{LCLS Schematic Overview}

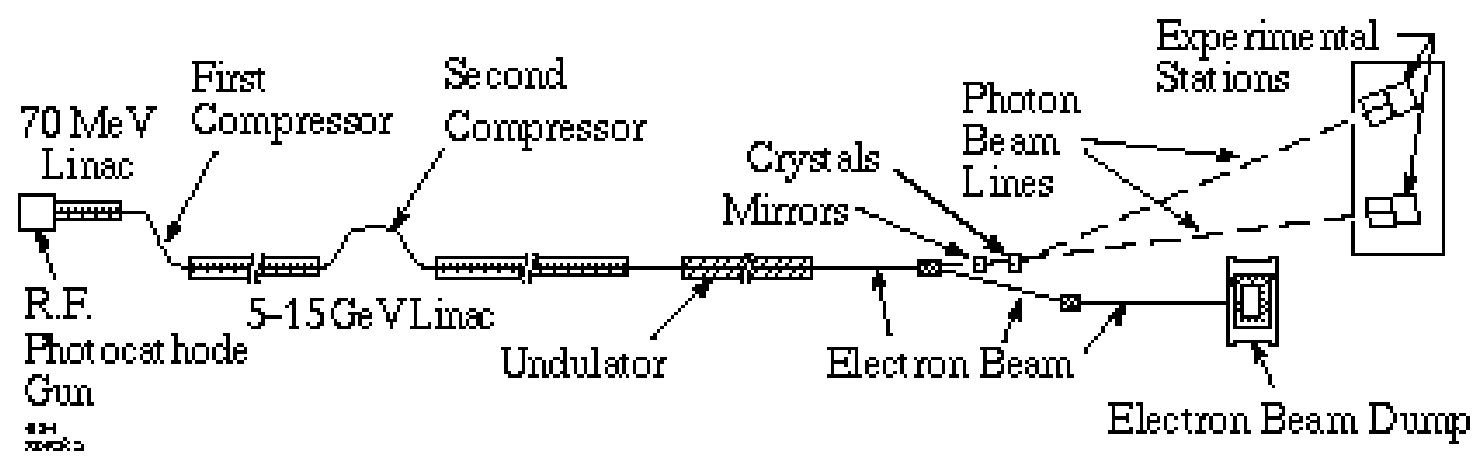


Fig. 9 


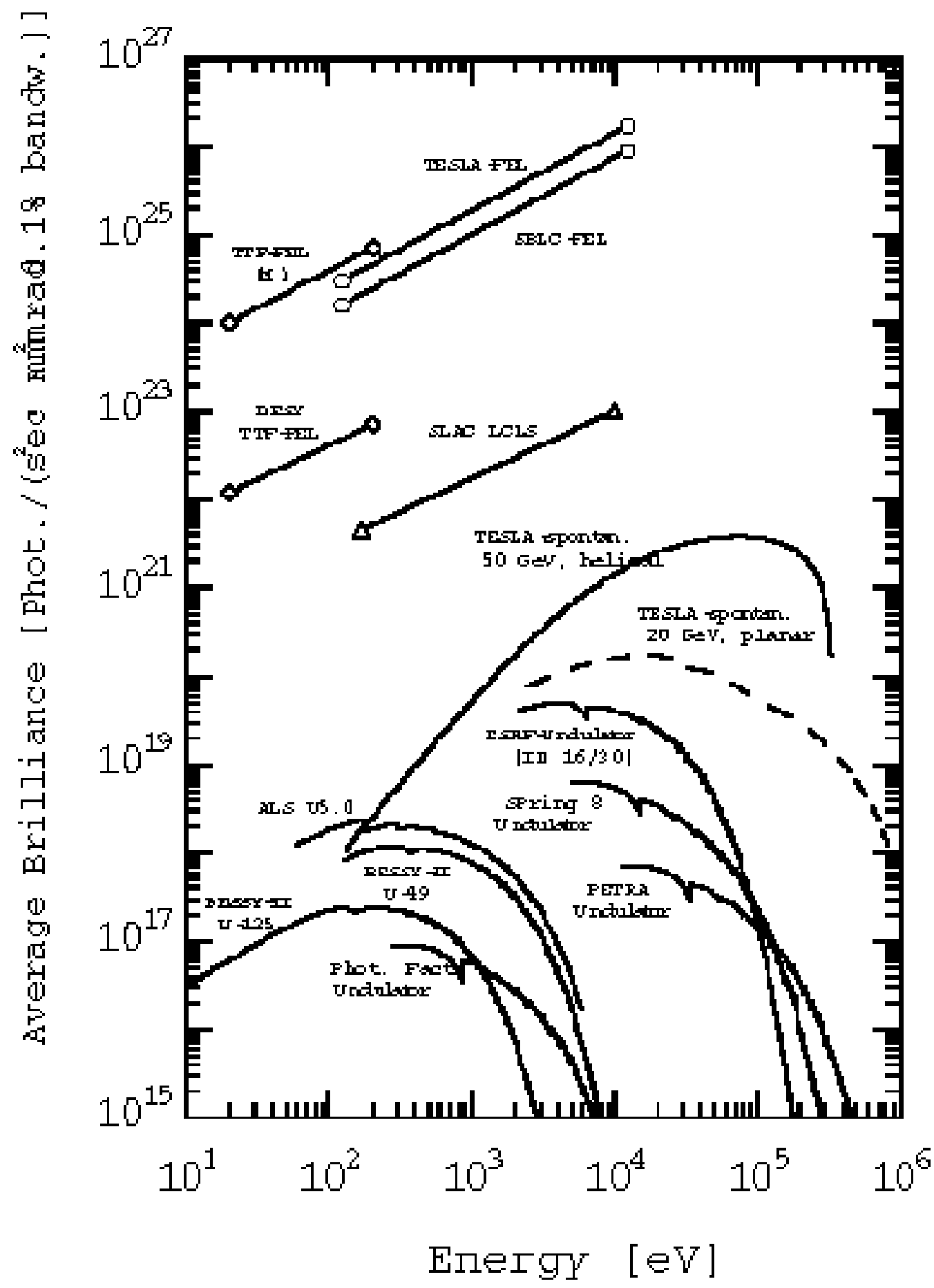


Fig. 10

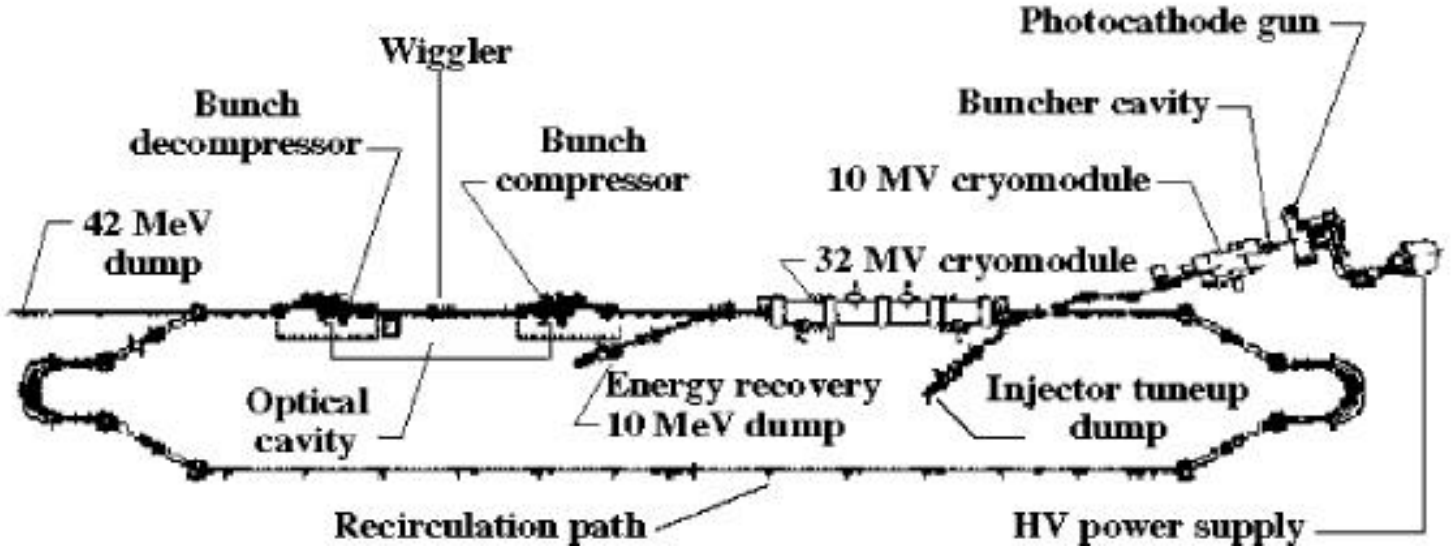

Fig. 11 